
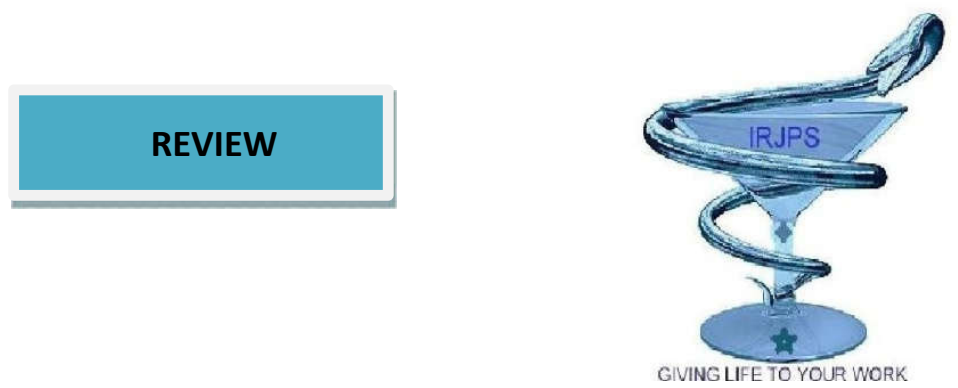

\title{
MEDICINE REGULATORY AUTHORITY (MRA) DIRECTORY OF COUNTRIES IN THE WORLD
}

\author{
J. Yoshasri", A. E. Prabahar, N. Ramarao \\ Chalapathi Institute Pharmaceutical Sciences, Lam, Guntur,India.
}

\section{Submitted on: 25.05.17; $\quad$ Revised on: 15.06.17; $\quad$ Accepted on: 28.06.17}

\begin{abstract}
:
All countries should /may have some kind of institution that regulates pharmaceuticals for human use . These institution are commonly referred to as MRA. The MRA is generally the institution responsible for the regulation and control of pharmaceuticals and other medical products within a country. In some countries the MRA is its own institutional unit, while on others it usually acts under the country's main public health institution, typically the Ministry of Health. Essential medicines are medicines that satisfy the priority health care needs of population. They are selected with regard to disease prevalence, safety, efficacy, and comparative cost effectiveness . They should be available at all times and at accessible prices. WHO publishes a model list of essential medicines that contains a core list and a complementary list. Each country is encouraged to prepare their own lists taking in to consideration local priorities. The latest WHO model EML can be found here.
\end{abstract}

KEY WORDS: MRA - Medicines Regulatory Authority, WHO - World Health Organization, EML Essential medicines list.

Corresponding Author: J. Yoshasri, Indian Research Journal of Pharmacy and Science; 13(2017) 972-1001 Journal Home Page: https://www.irjps.in E-mail: yoshasridhar@gmail.com DOI: 10.21276/irjps.2017.4.2.4 


\section{INTRODUCTION:}

Drug regulatory authority is an authority appointed by the government of a Member State to administer the granting of Marketing Authorization for pharmaceutical products in that country.

Drug regulation in the control of drug use by international agreement and / or by regulatory authorities such as the USFDA, the EMA and the Japanese Pharmaceutical and Medical Devices Agency.These authorities includes regulations concerned with the development, approval manufacturing and marketing of drugs.

A legal document issued by the competent medicine regulatory authority for the purpose of marketing or free distribution of product after evaluation for safety, efficacy and quality.
These regulatory authorities are different for different countries, and their websites and medical lists are different for each country. For to easy search this article includes their website link and their EML links and EML published years.

This article includes the lists of MRA of all ICP countries ,NON-ICP countries and includes direct links to their websites. Includes direct links to country's Essential Medicines List (EML) by region wise.

\section{AFRICA $^{1,2,12}$ :}

This includes all internationally recognized territories and states on the continent. The variation is a result of disputed territories and inconsistencies around the inclusion of island nations off the coast of Africa. The African Union recognizes $\mathbf{5 4}$ countries while the United Nations recognizes 54 as well.

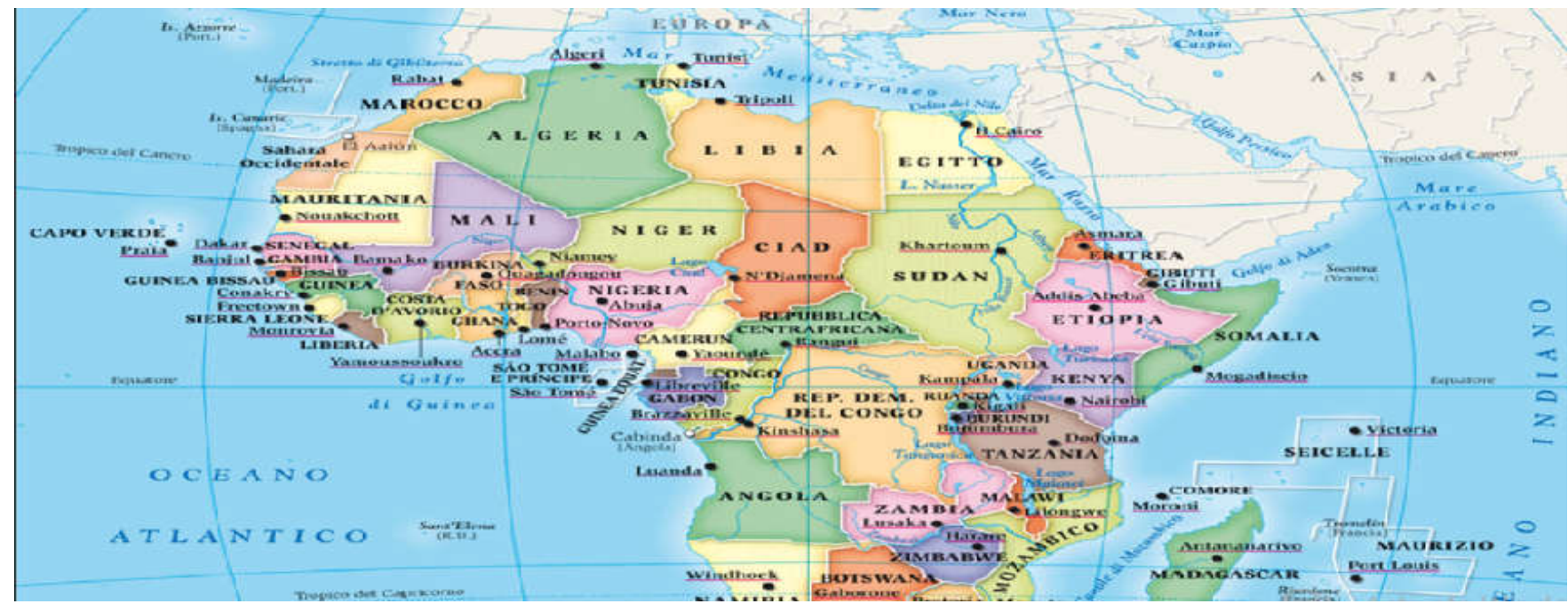

\begin{tabular}{|c|c|c|c|c|c|c|c|}
\hline SI.No & Region & $\begin{array}{c}\text { Countr } \\
y\end{array}$ & $\begin{array}{l}\text { Medicines } \\
\text { Agency/ } \\
\text { Authority } \\
\text { Acronym }\end{array}$ & $\begin{array}{l}\text { Medicines } \\
\text { Agency / } \\
\text { Authority }\end{array}$ & Website & $\begin{array}{c}\text { Essential } \\
\text { Medicines list }\end{array}$ & $\begin{array}{c}\text { Essenti } \\
\text { al } \\
\text { Medici } \\
\text { nes list } \\
\text { year }\end{array}$ \\
\hline 1 & Africa & Algeria & DPM & $\begin{array}{l}\text { Direction de } \\
\text { la pharmacie } \\
\text { et du } \\
\text { Medicament }\end{array}$ & $\begin{array}{l}\text { http://ands/pharmacie } \\
. \mathrm{med} / \mathrm{sommaire.htm}\end{array}$ & $\begin{array}{l}\frac{\text { http://www.who.int/ }}{\text { entity/selection }} \\
\text { medicines/country } \\
\underline{\text { lists/dza 2006.pdf }}\end{array}$ & 2006 \\
\hline
\end{tabular}

Ind Res J Pharm \& Sci|2017: June.:4(2) 


\begin{tabular}{|c|c|c|c|c|c|c|c|}
\hline 2 & Africa & Anglo & $\mathrm{MOH}$ & $\begin{array}{l}\text { Ministry of } \\
\text { Health }\end{array}$ & $\frac{\text { http://www.minsa.go }}{\underline{\text { v.ao/ }}}$ & $\begin{array}{c}\frac{\text { http://www.who.int/ }}{\text { entity/selection }} \\
\frac{\text { medicines/country }}{\text { lists/agoList }} \\
\text { 2008.pdf }\end{array}$ & 2008 \\
\hline 3 & Africa & Benin & & & & & \\
\hline 4 & Africa & $\begin{array}{c}\text { Botswa } \\
\text { na }\end{array}$ & $\mathrm{MOH}$ & $\begin{array}{l}\text { Ministry of } \\
\text { Health }\end{array}$ & $\frac{\text { http://www.moh.gov. }}{\underline{\text { bw/ }}}$ & $\begin{array}{l}\frac{\mathrm{http}: / / \text { apps.who.int/ }}{\text { medicinedocs/docu }} \\
\frac{\text { ments/s19479en/s19 }}{479 \mathrm{en} . \mathrm{pdf}}\end{array}$ & 2010 \\
\hline 5 & Africa & $\begin{array}{c}\text { Burkina } \\
\text { Faso }\end{array}$ & DGPML & $\begin{array}{l}\text { Direction } \\
\text { General of } \\
\text { Pharmacy, } \\
\text { Medicine } \\
\text { and } \\
\text { Pharmacy } \\
\text { Laboratories }\end{array}$ & $\frac{\text { http://www.dgpml.sa }}{\text { nte.gov.bf/ }}$ & $\frac{\frac{\text { http://www.remend. }}{\text { org/html/politique }}}{\frac{\text { pharmaceutique }}{\text { burki.html }}}$ & 2007 \\
\hline 6 & Africa & Burundi & & $\begin{array}{l}\text { Ministry of } \\
\text { Public } \\
\text { Health }\end{array}$ & $\frac{\underline{\mathrm{http}: / / \mathrm{www} \cdot m i n i s a n t e}}{. \mathrm{bi} /}$ & $\begin{array}{l}\frac{\text { http://www.who.int/ }}{\text { entity/selection }} \\
\frac{\text { medicines/country }}{\underline{\text { lists/bdi EML }}} \\
\text { 2009.pdf }\end{array}$ & 2009 \\
\hline 7 & Africa & $\begin{array}{c}\text { Camero } \\
\text { on }\end{array}$ & & $\begin{array}{l}\text { Ministry of } \\
\text { Public } \\
\text { Health }\end{array}$ & $\frac{\text { http://www.minisante }}{. \mathrm{cm} /}$ & $\begin{array}{l}\frac{\text { http://www.remend. }}{\text { org/Lmne Cameroun }} \\
\text { 2009.doc }\end{array}$ & 2009 \\
\hline 8 & Africa & $\begin{array}{l}\text { Cape } \\
\text { Verde }\end{array}$ & ARFA & $\begin{array}{c}\text { Agencia de } \\
\text { Regulacao e } \\
\text { Supervisao } \\
\text { dos Produtos } \\
\text { Farmaceutic } \\
\text { os e } \\
\text { Alimentares }\end{array}$ & $\frac{\text { http://www.arfa.cv/in }}{\underline{\text { dex.php }}}$ & $\begin{array}{l}\frac{\text { http://www.who.int/ }}{\text { entity/selection }} \\
\frac{\text { medicines/country }}{\text { lists/CaboVede2009. }} \\
\text { pdf }\end{array}$ & 2009 \\
\hline 9 & Africa & $\begin{array}{c}\text { Central } \\
\text { African } \\
\text { Rep }\end{array}$ & & $\begin{array}{c}\text { Direction } \\
\text { des services } \\
\text { Pharmaceuti } \\
\text { ques des } \\
\text { Laboratories } \\
\text { et de la } \\
\text { Medicine } \\
\text { Traditionnel } \\
\text { le }\end{array}$ & & $\begin{array}{l}\frac{\text { http://www.who.int/ }}{\text { entity/selection }} \\
\frac{\text { medicines/country }}{\underline{\text { lists/cafeml }}} \\
\underline{\text { 2009.pdf }}\end{array}$ & 2009 \\
\hline 10 & Africa & Chad & & $\begin{array}{l}\text { Ministry of } \\
\text { Public } \\
\text { Health: } \\
\text { Direction la } \\
\text { Pharmacie, } \\
\text { du } \\
\text { Medicament } \\
\text { et des }\end{array}$ & $\frac{\text { http://www.sante- }}{\text { tchand.org/ }}$ & $\begin{array}{l}\frac{\text { http://www.who.int/ }}{\text { entity/selection }} \\
\text { medicines/country } \\
\text { lists/Tchad 2007.pdf }\end{array}$ & 2007 \\
\hline & & & & & \multicolumn{2}{|c|}{ Ind Res J Pharm \& Sci|2017: June.:4(2) } & \\
\hline
\end{tabular}




\begin{tabular}{|c|c|c|c|c|c|c|c|}
\hline & & & & Laboratories & & & \\
\hline 11 & Africa & $\begin{array}{l}\text { Comoro } \\
\text { s, The }\end{array}$ & & $\begin{array}{l}\text { Direction } \\
\text { des } \\
\text { Laboratories } \\
\text { et des } \\
\text { Pharmacies } \\
\end{array}$ & & & \\
\hline 12 & Africa & $\begin{array}{l}\text { Congo, } \\
\text { Republi } \\
\text { c of the }\end{array}$ & & $\begin{array}{c}\text { Ministrere } \\
\text { de la Sante } \\
\text { Publique et } \\
\text { des Affairs } \\
\text { Sociales:Dir } \\
\text { ection des } \\
\text { Pharmacies, } \\
\text { du } \\
\text { Medicament } \\
\text { et des } \\
\text { Laboratories }\end{array}$ & & $\begin{array}{c}\frac{\text { http://www.who.int/ }}{\text { entity/selection }} \\
\frac{\text { medicines/country }}{\underline{\text { lists/cog nme }}} \\
\underline{2008 . p d f}\end{array}$ & 2008 \\
\hline 13 & Africa & $\begin{array}{l}\text { Coted'I } \\
\text { voire }\end{array}$ & $\overline{D P M}$ & $\begin{array}{l}\text { Directorate } \\
\text { of } \\
\text { Medicines } \\
\text { and } \\
\text { Pharmacy of } \\
\text { Cote } \\
\text { d'Ivoire } \\
\end{array}$ & $\frac{\text { http://www.dpmci.or }}{\mathrm{g} /}$ & $\begin{array}{l}\frac{\begin{array}{l}\text { http://www.who.int/ } \\
\text { entity/selection }\end{array}}{\text { medicines/country }} \\
\frac{\text { lists/LMECotedIVO }}{\underline{\text { IRE.pdf }}}\end{array}$ & \\
\hline 14 & Africa & $\begin{array}{l}\text { Congo, } \\
\text { Dem. } \\
\text { Rep. }\end{array}$ & DPM & $\begin{array}{l}\text { Ministere de } \\
\text { la Sante } \\
\text { Publique: } \\
\text { Direction de } \\
\text { la Pharmacie } \\
\text { et du } \\
\text { Medicament }\end{array}$ & $\begin{array}{c}\frac{\text { http://www.minisante }}{\text { rdc.cd/new/index.php }} \\
\frac{\text { /direction/76- }}{\text { direction-de-la- }} \\
\text { pharmacie-et-du- } \\
\text { medicament-dpm }\end{array}$ & $\frac{\frac{\text { http://www.apps.wh }}{\text { o.int.medicinedocs/e }}}{\frac{\text { n/m/abstract/Js1881 }}{\underline{7 \mathrm{fr} /}}}$ & 2010 \\
\hline 15 & Africa & Djibouti & & & & $\begin{array}{c}\frac{\text { http://www.who.int/ }}{\text { entity/selection }} \\
\frac{\text { medicines/country }}{\underline{\text { lists/dji }}} \\
\underline{\text { Liste 207.pdf }}\end{array}$ & 2007 \\
\hline 16 & Africa & Egypt & EDA & $\begin{array}{l}\text { Egyptian } \\
\text { Drug } \\
\text { Authority }\end{array}$ & $\frac{\text { http://www.eda.mohp }}{\text { gov.eg/ }}$ & $\begin{array}{c}\frac{\text { http://www.who.int/ }}{\text { entity/selection }} \\
\frac{\text { medicines/country }}{\text { lists/EGY EML }} \\
\frac{2006 . p d f}{2}\end{array}$ & 2006 \\
\hline 17 & Africa & $\begin{array}{l}\text { Equator } \\
\text { ial } \\
\text { Guinea } \\
\end{array}$ & & & & & \\
\hline 18 & Africa & Ethiopia & FMHACA & $\begin{array}{c}\text { Food, } \\
\text { Medicine } \\
\text { and Health } \\
\text { Care } \\
\text { Administrati } \\
\text { on and } \\
\text { control of } \\
\end{array}$ & $\begin{array}{c}\text { http://www.fmhaca.g } \\
\text { ov.et/ }\end{array}$ & $\begin{array}{c}\frac{\text { http://www.who.int/ }}{\text { entity/selection }} \\
\frac{\begin{array}{l}\text { medicines/country } \\
\text { lists/Ethiopia }\end{array}}{\underline{2010 . p d f}}\end{array}$ & 2010 \\
\hline & & & & & \multicolumn{2}{|c|}{ Ind Res J Pharm \& Sci|2017: June.:4(2) } & \\
\hline
\end{tabular}




\begin{tabular}{|c|c|c|c|c|c|c|c|}
\hline & & & & Ethiopia & & & \\
\hline 19 & Africa & Gabon & & $\begin{array}{l}\text { Direction } \\
\text { Medicament } \\
\text { et de la } \\
\text { Pharmacie }\end{array}$ & & $\frac{\frac{\text { http://www.remed.o }}{\text { rg/html/politique }}}{\frac{\text { pharmaceutique }}{\text { gabon.html }}}$ & No Year \\
\hline 20 & Africa & Gambia & & $\begin{array}{c}\text { Ministry of } \\
\text { Health and } \\
\text { Social } \\
\text { Welfare }\end{array}$ & $\frac{\text { http://www.moh.gov. }}{\mathrm{gm} /}$ & & \\
\hline 21 & Africa & Ghana & FDB & $\begin{array}{l}\text { Food and } \\
\text { Drug Board }\end{array}$ & $\frac{\text { http://www.fdbghana. }}{\text { gov.gh/ }}$ & $\begin{array}{l}\frac{\text { http://www.who.int/ }}{\text { entity/selection }} \\
\underline{\text { medicines/country }} \\
\underline{\text { lists/gha2004.pdf }}\end{array}$ & 2010 \\
\hline 22 & Africa & Guinea & & $\begin{array}{l}\text { Direction } \\
\text { Nationale de } \\
\text { la Pharmacie } \\
\text { et du } \\
\text { Laboratorie } \\
\end{array}$ & & & \\
\hline 23 & Africa & $\begin{array}{l}\text { Guinea- } \\
\text { Bissau }\end{array}$ & & & & & \\
\hline 24 & Africa & Kenya & & $\begin{array}{l}\text { Pharmacy } \\
\text { and Poisons } \\
\text { Board }\end{array}$ & $\frac{\text { http://www.pharmac }}{\text { yboardkenya. org/ }}$ & $\begin{array}{c}\frac{\text { http://apps.who.int/ }}{\underline{\text { medicinedoc }}} \\
\frac{\text { s/en/m/abstract/Js1 }}{\underline{8694 \mathrm{en} /}}\end{array}$ & 2010 \\
\hline 25 & Africa & Lesotho & & & & $\begin{array}{l}\text { http://www.who.int/ } \\
\text { entity/select } \\
\text { ion_medicines/coun } \\
\text { try_lists/lso } \\
\text { 2005_STGs_EML.p } \\
\text { df }\end{array}$ & 2005 \\
\hline 26 & Africa & Liberia & & $\begin{array}{c}\text { Liberia } \\
\text { Medicin } \\
\text { es and } \\
\text { Health } \\
\text { Product } \\
\text { s } \\
\text { Regulat } \\
\text { ory } \\
\text { Authority }\end{array}$ & & & \\
\hline 27 & Africa & $\begin{array}{c}\text { Madaga } \\
\text { scar }\end{array}$ & & $\begin{array}{l}\text { Ministry of } \\
\text { Public } \\
\text { Health }\end{array}$ & $\frac{\text { http://www.sante.gov }}{\underline{\text { mg/index.ph p }}}$ & $\begin{array}{l}\frac{\text { http://www.remed.o }}{\mathrm{rg} / \mathrm{html} / \mathrm{poli}} \\
\text { tique pharmaceutiq } \\
\underline{\text { ue madag.ht } \underline{\mathrm{ml}}}\end{array}$ & No Year \\
\hline 28 & Africa & Malawi & & $\begin{array}{l}\text { Ministry of } \\
\text { Health: } \\
\text { Directorate } \\
\text { of Health }\end{array}$ & $\begin{array}{c}\text { http://www.malawi.g } \\
\text { ov.mw/index. } \\
\text { php?option=com_co } \\
\text { ntent\&view=ar }\end{array}$ & & \\
\hline & & & & & \multicolumn{2}{|c|}{ Ind Res J Pharm \& Sci|2017: June.:4(2) } & 76 \\
\hline
\end{tabular}




\begin{tabular}{|c|c|c|c|c|c|c|c|}
\hline & & & & $\begin{array}{l}\text { Technical } \\
\text { Support } \\
\text { Services }\end{array}$ & $\begin{aligned} \underline{\text { ticle\&id }}=55 \& \text { Itemid } \\
\\
=85\end{aligned}$ & & \\
\hline 29 & Africa & Mali & & $\begin{array}{l}\text { Ministry of } \\
\text { Health: } \\
\text { Department } \\
\text { of Pharmacy } \\
\text { \&Medicame } \\
\text { nt }\end{array}$ & $\begin{array}{l}\frac{\text { http://www.sante.go }}{\text { v.ml/index.ph }} \\
\text { p?option=com_conte } \\
\underline{\text { nt\&task }=\text { view }} \\
\text { \&id=31\&Itemid }=72\end{array}$ & $\begin{array}{l}\frac{\text { http://www.who.int/ }}{\text { entity/select }} \\
\frac{\text { ion_medicines/coun }}{\text { try lists/mli }} \\
\underline{\text { EML_2008.pdf }}\end{array}$ & 2008 \\
\hline 30 & Africa & $\begin{array}{c}\text { Maurita } \\
\text { nia }\end{array}$ & $\mathrm{MOH}$ & $\begin{array}{l}\text { Ministry of } \\
\text { Health: } \\
\text { Pharmacy } \\
\text { and } \\
\text { Laboratory }\end{array}$ & 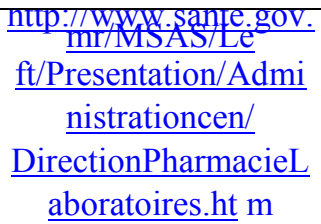 & $\begin{array}{l}\frac{\mathrm{http}_{\text {//apps.who.int/ }}}{\text { medicinedoc }} \\
\frac{\text { s/documents/s17813 }}{\underline{\text { fr/s17813fr. } \mathrm{pdf}}}\end{array}$ & 2007 \\
\hline 31 & Africa & $\begin{array}{c}\text { Mauriti } \\
\text { us }\end{array}$ & & $\begin{array}{l}\text { Ministry of } \\
\text { Health and } \\
\text { Quality of } \\
\text { Life } \\
\end{array}$ & $\begin{array}{c}\underline{\text { http://www.gov.mu/p }} \\
\text { ortal/site/mo hsite }\end{array}$ & & \\
\hline 32 & Africa & $\begin{array}{c}\text { Morocc } \\
\text { o }\end{array}$ & $\mathrm{MOH}$ & $\begin{array}{l}\text { Ministry of } \\
\text { Health }\end{array}$ & $\begin{array}{c}\frac{\text { http://srvweb.sante.g }}{\text { ov.ma/Medic }} \\
\frac{\text { aments/Pages/defa }}{\text { ult.aspx }}\end{array}$ & $\begin{array}{l}\frac{\text { http://www.who.int/ }}{\frac{\text { entity/select }}{\text { medicines/coun }}} \\
\underline{\text { ion_try lists/mar }} \\
\text { LME 2008.pdf }\end{array}$ & 2008 \\
\hline 33 & Africa & $\begin{array}{l}\text { Mozam } \\
\text { bique }\end{array}$ & $\mathrm{MOH}$ & $\begin{array}{l}\text { Ministry of } \\
\text { Health }\end{array}$ & $\frac{\underline{\mathrm{http}: / / \text { www.misau.go }}}{\underline{\underline{\mathrm{v} . \mathrm{mz} / \mathrm{pt} / \mathrm{medi}}}} \frac{\underline{\text { camentos }}}{\underline{ }}$ & & \\
\hline 34 & Africa & $\begin{array}{c}\text { Namibi } \\
\text { a }\end{array}$ & NMRC & $\begin{array}{l}\text { Namibia } \\
\text { Medicines } \\
\text { Regulatory } \\
\text { Authority }\end{array}$ & $\frac{\text { http://www.nmrc.c }}{\underline{\text { om.na/ }}}$ & $\begin{array}{l}\frac{\text { http://www.who.int/ }}{\text { entity/select }} \\
\frac{\text { ion_medicines/coun }}{\text { try lists/nam }} \\
\text { eml 2008.pdf }\end{array}$ & 2008 \\
\hline 35 & Africa & Niger & & $\begin{array}{l}\text { Ministry of } \\
\text { Public } \\
\text { Health }\end{array}$ & $\frac{\frac{\text { http://www.gouv.ne/i }}{\text { ndex.php?id }}}{\frac{\text { page }=}{\underline{30}}}$ & & \\
\hline 36 & Africa & Nigeria & NAFDAC & $\begin{array}{l}\text { National } \\
\text { Agency for } \\
\text { Food and } \\
\text { Drug } \\
\text { Administrati } \\
\text { on and } \\
\text { Control }\end{array}$ & $\begin{array}{c}\text { http://www.nafdac. } \\
\text { gov.ng/ }\end{array}$ & & \\
\hline 37 & Africa & Rwanda & MOA & $\begin{array}{l}\text { Ministry of } \\
\text { Health }\end{array}$ & $\begin{array}{c}\text { http://www.moh.g } \\
\text { ov.rw/ }\end{array}$ & $\begin{array}{l}\text { http://www.who.int/ } \\
\text { entity/select } \\
\text { ion_medicines/count }\end{array}$ & 2010 \\
\hline
\end{tabular}




\begin{tabular}{|c|c|c|c|c|c|c|c|}
\hline & & & & & & $\frac{\text { ry lists/RW }}{\text { A LNME 2010.pdf }}$ & \\
\hline 38 & Africa & $\begin{array}{c}\text { Sao } \\
\text { Tome } \\
\& \text { Princi } \\
\text { pe }\end{array}$ & & & & & \\
\hline 39 & Africa & Senegal & & $\begin{array}{l}\text { Direction de } \\
\text { la Pharmacie } \\
\text { et des } \\
\text { Laboratories }\end{array}$ & $\begin{array}{c}\frac{\text { http://www.sante.gou }}{\text { v.sn/index.p }} \\
\text { hp?option=com_cont } \\
\text { ent\&view=arti } \\
\text { cle\&id }=1727\end{array}$ & $\begin{array}{l}\frac{\text { http://www.who.int/ }}{\text { entity/select }} \\
\text { ion medicines/coun } \\
\text { try_lists/sen } \\
\text { eml 2008.pdf }\end{array}$ & 2008 \\
\hline 40 & Africa & $\begin{array}{c}\text { Seychel } \\
\text { les }\end{array}$ & $\mathrm{MOH}$ & $\begin{array}{l}\text { Ministry of } \\
\text { Health }\end{array}$ & $\begin{array}{l}\frac{\mathrm{http} / / \text { www.health.go }}{\text { v.sc/index.ph }} \\
\frac{\text { p?option=com conte }}{\text { nt\&view=articl }} \\
\frac{\underline{\text { e\&id }=255 \& \text { Itemid }}=2}{\underline{60}}\end{array}$ & $\begin{array}{l}\frac{\text { http://www.who.int/ }}{\text { entity/select }} \\
\frac{\text { ion medicines/coun }}{\text { try lists/Seyc }} \\
\underline{\text { helles2010.pdf }}\end{array}$ & 2010 \\
\hline 41 & Africa & $\begin{array}{l}\text { Sierra } \\
\text { Leone }\end{array}$ & & $\begin{array}{l}\text { Ministry of } \\
\text { Health and } \\
\text { Sanitation }\end{array}$ & $\begin{array}{c}\frac{\text { http://www.health.go }}{\mathrm{v.sl} / \text { home/re }} \\
\begin{array}{c}\text { gulatory- } \\
\text { bodies/pharmacy- }\end{array} \\
\text { board }\end{array}$ & & \\
\hline 42 & Africa & $\begin{array}{l}\text { South } \\
\text { Africa }\end{array}$ & MCC & $\begin{array}{l}\text { Medicines } \\
\text { Control } \\
\text { Council }\end{array}$ & $\frac{\text { http://www.mecza }}{. \mathrm{com} /}$ & $\begin{array}{l}\frac{\text { http://www.who.int/ }}{\text { entity/select }} \\
\text { ion_medicines/coun } \\
\text { try_lists/zaf_ } \\
\text { adult 2006.pdf }\end{array}$ & 2006 \\
\hline 43 & Africa & Sudan & NMPB & $\begin{array}{c}\text { National } \\
\text { Medicines } \\
\text { and Poisons } \\
\text { Board }\end{array}$ & $\frac{\text { http://www.nmpb. }}{\text { gov.sd/ }}$ & $\begin{array}{l}\frac{\text { http://www.who.int/ }}{\text { entity/select }} \\
\frac{\text { ion medicines/coun }}{\text { try lists/sdn }} \\
\text { eml 2007.pdf }\end{array}$ & 2007 \\
\hline 44 & Africa & $\begin{array}{c}\text { Swazila } \\
\text { nd }\end{array}$ & $\mathrm{MOH}$ & $\begin{array}{l}\text { Ministry of } \\
\text { Health }\end{array}$ & $\begin{array}{l}\frac{\text { http: } / / \text { www.gov.sz/in }}{\text { dex.php?opti }} \\
\frac{\text { on=com_content } \& v i}{\text { cow }=\text { article\&id }=} \\
\frac{267 \& \text { Itemid }=40}{3}\end{array}$ & & \\
\hline 45 & Africa & $\begin{array}{c}\text { Tanzani } \\
\text { a }\end{array}$ & TFDA & $\begin{array}{l}\text { Tanzania } \\
\text { Food and } \\
\text { Drug } \\
\text { Authority }\end{array}$ & $\frac{\text { http://www.tfda.o }}{\underline{\text { r.tz/ }}}$ & $\begin{array}{l}\frac{\text { http://www.who.int/ }}{\text { entity/select }} \\
\underline{\text { ion medicines/coun }} \\
\text { try_lists/tza} \\
\text { stg_neml_2007.pdf }\end{array}$ & 2007 \\
\hline 46 & Africa & Togo & & & & $\begin{array}{l}\frac{\text { http://www.remed.o }}{\text { rg/html/poli }} \\
\frac{\text { tique pharmaceutiq }}{\text { ue togo.html }}\end{array}$ & No Year \\
\hline
\end{tabular}




\begin{tabular}{|c|c|c|c|c|c|c|c|}
\hline 47 & Africa & Tunisia & & $\begin{array}{l}\text { Ministry of } \\
\text { Public } \\
\text { Health }\end{array}$ & $\frac{\mathrm{http}: / / \mathrm{www} \cdot \mathrm{dpm} .}{\underline{\mathrm{tn} /}}$ & $\begin{array}{l}\frac{\frac{\text { http://www.who.int/ }}{\text { entity/select }}}{\frac{\underline{\text { ion medicines/coun }}}{\text { try lists/TUN }}} \\
\frac{\text { 2e FORMU }}{\text { LAIRE.pdf }}\end{array}$ & 2008 \\
\hline 48 & Africa & Uganda & NDA & $\begin{array}{l}\text { National } \\
\text { Drug } \\
\text { Authority }\end{array}$ & $\frac{\text { http://www.nda.o }}{\underline{\text { r.ug/ }}}$ & $\begin{array}{l}\frac{\text { http://www.who.int/ }}{\text { entity/select }} \\
\frac{\text { ion_medicines/coun }}{\text { try lists/uga }} \\
\frac{\text { eml_200 }}{7 . \mathrm{pdf}}\end{array}$ & 2007 \\
\hline 49 & Africa & Zambia & $\overline{\mathrm{PRA}}$ & $\begin{array}{l}\text { Pharmaceuti } \\
\text { cal } \\
\text { Regulatory } \\
\text { Authority } \\
\end{array}$ & $\frac{\text { http://www.pra.go }}{\underline{\text { v.zm/ }}}$ & & \\
\hline 50 & Africa & $\begin{array}{c}\text { Zimbab } \\
\text { we }\end{array}$ & MCAZ & $\begin{array}{l}\text { Medicines } \\
\text { Control } \\
\text { Authority of } \\
\text { Zimbabwe }\end{array}$ & $\frac{\underline{\text { http://www.mcaz. }}}{\underline{\text { co.zw/ }}}$ & $\begin{array}{l}\frac{\frac{\text { http://www.who.int/ }}{\text { entity/select }}}{\text { ion medicines/count }} \\
\frac{\text { ry lists/EDLI }}{\text { Z06.pdf }}\end{array}$ & 2006 \\
\hline
\end{tabular}

\section{ASIA \& PACIFIC ${ }^{1,2,13,14}$ :}

Being the biggest continent in the world, Asia includes 50 independent countries and occupies the eastern part of the single Eurasian landmass. Surrounded by the Arctic Ocean from the north, by the Pacific Ocean from the east and by the Indian Ocean from the south, it is separated from Africa by Suez Canal. The Mediterranean Sea and Black Sea separate Asia from Europe, farther the overland border runs through the Caucasus Mountains, the Caspian Sea, the Ural River and Ural Mountains. This boundary crosses through the territory of Russia, Kazakhstan, Azerbaijan, Georgia and Turkey, so these states are in both continents.

Armenia and Cyprus geographically are in Asia, but politically and culturally they also are considered as a part of Europe.

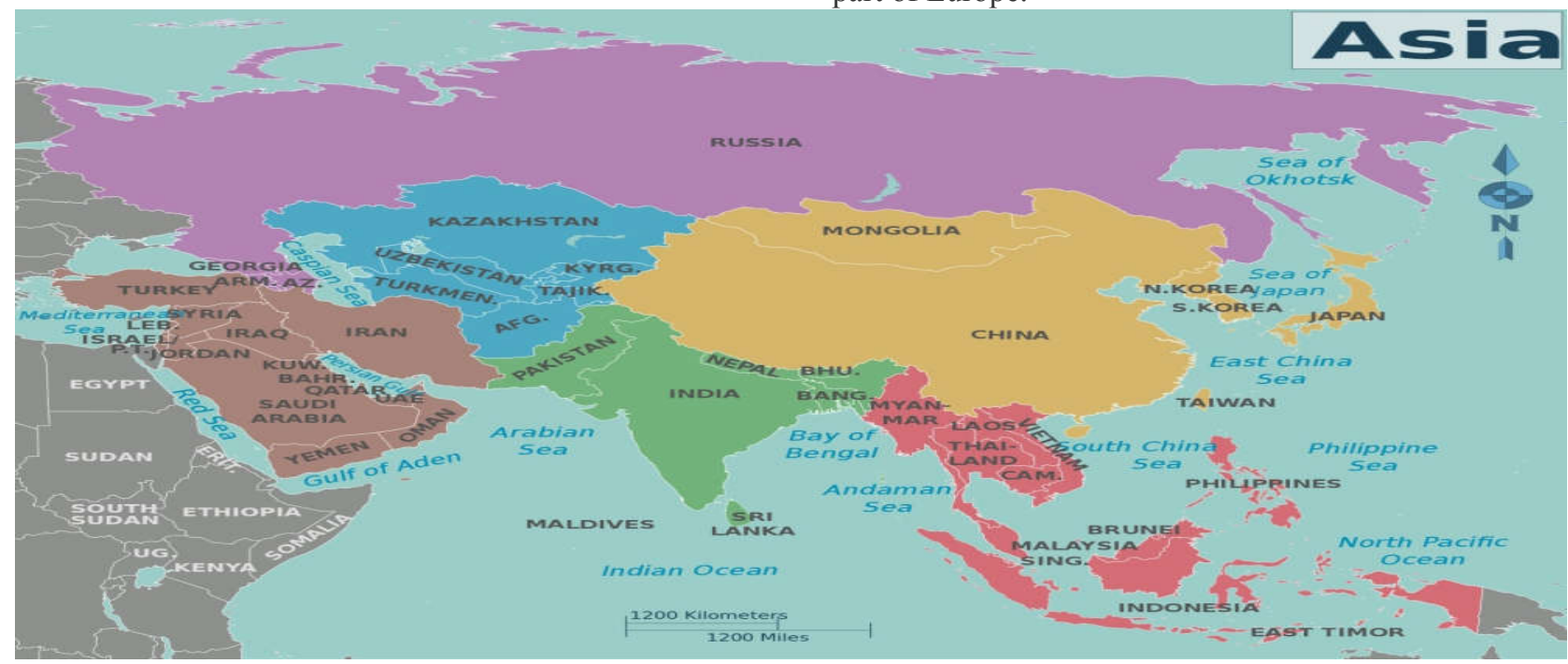




\begin{tabular}{|c|c|c|c|c|c|c|c|}
\hline $\begin{array}{l}\text { Sr. } \\
\text { No }\end{array}$ & $\begin{array}{c}\text { Regio } \\
\text { n }\end{array}$ & Country & 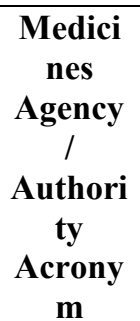 & $\begin{array}{c}\text { Medicines Agency / } \\
\text { Authority }\end{array}$ & Website & $\begin{array}{c}\text { Essential } \\
\text { Medicines list }\end{array}$ & $\begin{array}{c}\text { Essen } \\
\text { tial } \\
\text { Medi } \\
\text { cines } \\
\text { list } \\
\text { year }\end{array}$ \\
\hline 1 & $\begin{array}{c}\text { Asia } \\
\& \\
\text { Pacific }\end{array}$ & $\begin{array}{c}\text { Banglades } \\
\mathrm{h}\end{array}$ & DGDA & $\begin{array}{c}\text { Directorate General of Drug } \\
\text { Administration }\end{array}$ & $\frac{\text { http://www.dgda. }}{\text { gov.bd/ }}$ & & 2008 \\
\hline 2 & $\begin{array}{c}\text { Asia } \\
\& \\
\text { Pacific }\end{array}$ & Bhutan & $\overline{\text { DRA }}$ & Drug Regulatory Authority & $\frac{\text { http://ww.dra.go }}{\text { v.bt/ }}$ & $\begin{array}{l}\frac{\mathrm{http}: / / \text { apps.who.int/ }}{\text { medicinedocs/docu }} \\
\frac{\text { ments/s19471en/s1 }}{9471 \mathrm{en} . \mathrm{pdf}}\end{array}$ & 2012 \\
\hline 3 & $\begin{array}{c}\text { Asia } \\
\& \\
\text { Pacific }\end{array}$ & Brunei & DRS & Drug Registration Section & $\begin{array}{l}\text { http://www.moh. } \\
\text { gov.bn/pharmacy } \\
\text { services/drudregi } \\
\text { stration.htm }\end{array}$ & & \\
\hline 4 & $\begin{array}{c}\text { Asia } \\
\& \\
\text { Pacific }\end{array}$ & Cambodia & & & & $\begin{array}{l}\frac{\text { http://www.who.int }}{\text { /entity/selection }} \\
\frac{\text { medicines/country }}{\text { lists/khm }} \\
\frac{\text { essentialdrugs }}{\text { 2003.pdf }}\end{array}$ & \\
\hline 5 & $\begin{array}{c}\text { Asia } \\
\& \\
\text { Pacific }\end{array}$ & $\begin{array}{l}\text { China, } \\
\text { People's } \\
\text { Republic } \\
\text { of }\end{array}$ & SFDA & $\begin{array}{c}\text { State Food Drug } \\
\text { Administration // // } \\
\text { National System of } \\
\text { Essential Medicines } \\
\text { (NSEM) (part of the } \\
\text { MOH) }\end{array}$ & $\begin{array}{l}\text { http://eng.sfd } \\
\text { a.gov.cn/ }\end{array}$ & 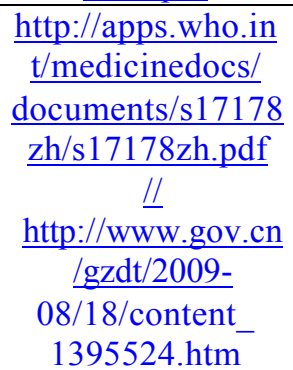 & 2009 \\
\hline 6 & $\begin{array}{c}\text { Asia } \\
\& \\
\text { Pacific }\end{array}$ & Fiji & IRA & $\begin{array}{c}\text { Inspectorate \& Regulatory } \\
\text { Authority }\end{array}$ & $\begin{array}{c}\frac{\text { http://www.healt }}{\underline{\text { h.gov.fj/ira.html }}} \\
\frac{\#}{\frac{\text { T7fIR }}{8 \text { Wzn }}} \\
\frac{\text { Xo }}{2}\end{array}$ & $\begin{array}{l}\frac{\text { http://www.who.i }}{\text { nt/entity/selectio }} \\
\underline{\text { n_medicines/coun }} \\
\frac{\text { try lists/fji formu }}{\text { lary_2006.pdf }}\end{array}$ & 2006 \\
\hline 7 & $\begin{array}{c}\text { Asia } \\
\& \\
\text { Pacific }\end{array}$ & $\begin{array}{l}\text { Hong } \\
\text { Kong, } \\
\text { China }\end{array}$ & & $\begin{array}{c}\text { Department of Health: Drug } \\
\text { Office }\end{array}$ & $\begin{array}{c}\frac{\text { http://www.psdh }}{\text { gov.hk/eps/root }} \\
\frac{l}{\text { en/le }} \\
\frac{\text { vel.h }}{\text { tml }}\end{array}$ & & \\
\hline 8 & $\begin{array}{c}\text { Asia } \\
\&\end{array}$ & India & CDSCO & Central Drugs Standard & http://cdsco. & $\frac{\text { http://www.who.i }}{\text { nt/entity/selectio }}$ & 2011 \\
\hline
\end{tabular}




\begin{tabular}{|c|c|c|c|c|c|c|c|}
\hline & Pacific & & & Control Organization & nic.in/ & $\begin{array}{l}\text { n_medicines/coun } \\
\text { try_lists/India_N } \\
\text { L ME 2011.pdf }\end{array}$ & \\
\hline 9 & $\begin{array}{c}\text { Asia } \\
\& \\
\text { Pacific }\end{array}$ & Indonesia & $\begin{array}{l}\text { NA- } \\
\text { DFC }\end{array}$ & $\begin{array}{c}\text { The National Agency of Drug } \\
\text { and Food Control }\end{array}$ & $\frac{\text { http://www.p }}{\text { om.go.id/ }}$ & 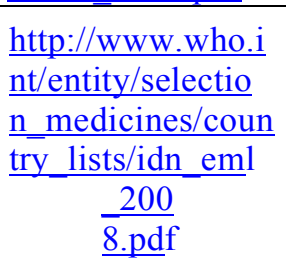 & 2008 \\
\hline 10 & $\begin{array}{c}\text { Asia } \\
\& \\
\text { Pacific }\end{array}$ & Lao PDR & & & & & \\
\hline 11 & $\begin{array}{c}\text { Asia } \\
\& \\
\text { Pacific }\end{array}$ & $\begin{array}{l}\text { Macao, } \\
\text { China }\end{array}$ & & & & & \\
\hline 12 & $\begin{array}{c}\text { Asia } \\
\& \\
\text { Pacific }\end{array}$ & Malaysia & $\begin{array}{l}\text { MPCB- } \\
\text { MOH }\end{array}$ & $\begin{array}{c}\text { National Pharmaceutical } \\
\text { Control Bureau - MOH } \\
\text { Malaysia }\end{array}$ & $\begin{array}{l}\frac{\text { http://www.porta }}{\text { l.bpfk.gov.my/in }} \\
\text { dex.cfm; } \\
\text { http://www.phar } \\
\text { macy.gov.my/ind } \\
\text { ex.cfm }\end{array}$ & $\begin{array}{l}\frac{\text { http://www.who.i }}{\text { nt/entity/selectio }} \\
\frac{\text { n_medicines/coun }}{\text { try_lists/mys ned }} \\
\frac{1 \text { 2008.pdf }}{200}\end{array}$ & 2008 \\
\hline 13 & $\begin{array}{c}\text { Asia } \\
\& \\
\text { Pacific }\end{array}$ & Maldives & & $\begin{array}{c}\text { Maldives Food and Drug } \\
\text { Authority }\end{array}$ & & $\frac{\frac{\text { http://apps.who.in }}{\text { t/medicinedocs/ }}}{\frac{\text { documents/s1704 }}{\text { 4e/s17044e.pdf }}}$ & 2009 \\
\hline 14 & $\begin{array}{c}\text { Asia } \\
\& \\
\text { Pacific }\end{array}$ & Mongolia & & Ministry of Health & $\begin{array}{l}\frac{\text { http://www.moh. }}{\mathrm{mn} / ;} \\
\frac{\mathrm{http} / / \mathrm{www} \cdot \mathrm{ssia} .}{\text { gov.mn/ }}\end{array}$ & $\frac{\frac{\text { http://apps.who.in }}{\text { t/medicinedocs/ }}}{\frac{\text { documents/s1954 }}{\text { dmn/s19547mn.p }}}$ & 2009 \\
\hline 15 & $\begin{array}{c}\text { Asia } \\
\& \\
\text { Pacific }\end{array}$ & Myanmar & & & & $\begin{array}{l}\frac{\text { http://www.who.i }}{\text { nt/entity/selectio }} \\
\frac{\text { n_medicines/coun }}{\text { try_lists/mmr_em }} \\
\underline{1 . p d f}\end{array}$ & \\
\hline 16 & $\begin{array}{c}\text { Asia } \\
\& \\
\text { Pacific }\end{array}$ & Nepal & DDA & $\begin{array}{l}\text { Department of Drug } \\
\text { Administration }\end{array}$ & $\begin{array}{l}\text { http://www.dda.g } \\
\text { ov.np/req moder } \\
\text { n_medicine.php }\end{array}$ & $\frac{\frac{\mathrm{http} / / \text { www.who.i }}{\mathrm{nt} / \text { entity/selectio }}}{\frac{\mathrm{n} \text { medicines/coun }}{\text { try_lists/npl_eml }}}$ & 2009 \\
\hline 17 & $\begin{array}{c}\text { Asia } \\
\& \\
\text { Pacific }\end{array}$ & Pakistan & $\begin{array}{c}\text { DCOM } \\
\text { OH }\end{array}$ & $\begin{array}{l}\text { Drugs Control } \\
\text { Organization, Ministry } \\
\text { of Health }\end{array}$ & & $\begin{array}{c}\frac{\text { http://www.who.i }}{\text { nt/entity/selectio }} \\
\frac{\text { n medicines/coun }}{\text { try lists/pak_EM }} \\
\underline{L} \\
\underline{200}\end{array}$ & 2007 \\
\hline
\end{tabular}




\begin{tabular}{|c|c|c|c|c|c|c|c|}
\hline & & & & & & 7.pdf & \\
\hline 18 & $\begin{array}{l}\text { Asia } \\
\& \\
\text { Pacific }\end{array}$ & $\begin{array}{l}\text { Philippine } \\
\text { s }\end{array}$ & FDA & $\begin{array}{l}\text { Food and Drug } \\
\text { Administration }\end{array}$ & $\frac{\text { http://www.f }}{\text { da.gov.ph/ }}$ & $\begin{array}{l}\frac{\mathrm{http} / / \text { www.who.i }}{\mathrm{nt} / \text { entity/selectio }} \\
\underline{\text { n_medicines/coun }} \\
\text { try lists/Phillipine } \\
\underline{\text { sEML2008.pdf }}\end{array}$ & 2008 \\
\hline 19 & $\begin{array}{c}\text { Asia } \\
\& \\
\text { Pacific }\end{array}$ & Singapore & HAS & $\begin{array}{c}\text { Health Sciences } \\
\text { Authority }\end{array}$ & $\frac{\text { http://www.h }}{\underline{\text { sa.gov.sg// }}}$ & & \\
\hline 20 & $\begin{array}{c}\text { Asia } \\
\& \\
\text { Pacific }\end{array}$ & Sri Lanka & $\overline{\text { DRA }}$ & $\begin{array}{l}\text { Drug Regulatory } \\
\text { Authority of Sri } \\
\text { Lanka }\end{array}$ & $\begin{array}{r}\underline{\text { http://203.94.76 }} \\
\frac{.60 / \mathrm{DR}}{\mathrm{A} / \mathrm{hom}} \\
\underline{\text { e.ht } \underline{\mathrm{m}}}\end{array}$ & $\begin{array}{l}\underline{\mathrm{http}: / / \text { www.who.i }} \\
\text { nt/entity/selectio } \\
\text { n_medicines/coun } \\
\text { try lists/lka_eml } \\
\text { 2009.pdf }\end{array}$ & 2009 \\
\hline 21 & $\begin{array}{c}\text { Asia } \\
\& \\
\text { Pacific }\end{array}$ & $\begin{array}{l}\text { Taipei, } \\
\text { China }\end{array}$ & FDA & $\begin{array}{c}\text { Food and Drug } \\
\text { Administration, Dept. of } \\
\text { Health }\end{array}$ & 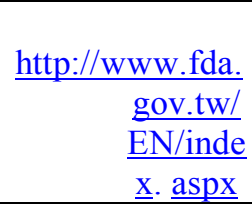 & & \\
\hline 22 & $\begin{array}{c}\text { Asia } \\
\& \\
\text { Pacific }\end{array}$ & Thailand & FDA & $\begin{array}{c}\text { Food and Drug } \\
\text { Administration, Ministry } \\
\text { of Public Health }\end{array}$ & $\begin{array}{r}\text { http://www.fda. } \\
\frac{\text { moph.go }}{\underline{\text { th/eng/i }}} \\
\underline{\underline{n}} \\
\underline{\text { dex.stm }}\end{array}$ & $\begin{array}{l}\frac{\text { http://www.who.i }}{\text { nt/entity/selectio }} \\
\underline{\text { n_medicines/coun }} \\
\underline{\text { try_lists/tha_nedl }} \\
\frac{200}{8 . \mathrm{pdf}}\end{array}$ & 2008 \\
\hline 23 & $\begin{array}{c}\text { Asia } \\
\& \\
\text { Pacific }\end{array}$ & Vietnam & DAV & $\begin{array}{c}\text { Drug Administration } \\
\text { of Vietnam }\end{array}$ & $\frac{\text { http://www.d }}{\underline{\text { av.gov.vn/ }}}$ & $\begin{array}{l}\frac{\mathrm{http}: / / \text { apps.who.in }}{\mathrm{t} / \text { medicinedocs/ }} \\
\text { documents/s1953 } \\
\text { 2vi/s19532vi.pdf }\end{array}$ & 2008 \\
\hline
\end{tabular}

$\begin{aligned} & \text { COMMON WEALTH OF INDEPENDENT } \\ & \text { STATES }{ }^{1-4} \text { : }\end{aligned}$
$\begin{aligned} & \text { of the Soviet Union. Nine out of the 15 former Soviet } \\ & \text { Republics are member states, and two are associate } \\ & \text { members } \\ & \text { (Ukraine and Turkmenistan). Georgia withdrew its }\end{aligned}$
$\begin{aligned} & \text { The Commonwealth of Independent States, also } \\ & \text { called the Russian Commonwealth (to distinguish it } \\ & \text { from the British Commonwealth of Nations), is } \\ & \text { a regional organization formed during the dissolution }\end{aligned}$
$\begin{aligned} & \text { states (Estonia, Lithuania and Latvia) chose not to } \\ & \text { participate. }\end{aligned}$

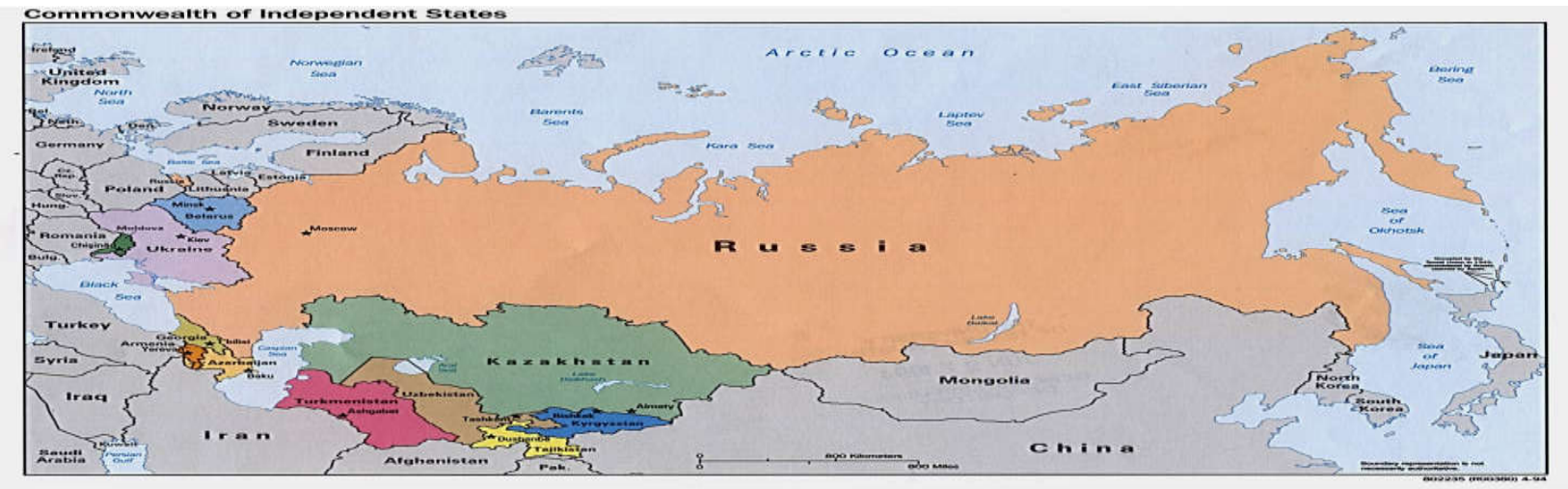




\begin{tabular}{|c|c|c|c|c|c|c|c|}
\hline $\begin{array}{l}\text { Sr. } \\
\text { No }\end{array}$ & Region & Country & $\begin{array}{c}\text { Medicines } \\
\text { Agency/ } \\
\text { Authority } \\
\text { Acronym }\end{array}$ & $\begin{array}{l}\text { Medicines Agency / } \\
\text { Authority }\end{array}$ & Website & $\begin{array}{c}\text { Essential } \\
\text { Medicines list }\end{array}$ & $\begin{array}{c}\text { Essential } \\
\text { Medicin } \\
\text { es list } \\
\text { year }\end{array}$ \\
\hline 1 & $\begin{array}{l}\text { Common } \\
\text { wealth of } \\
\text { Independen } \\
t \text { States }\end{array}$ & Armenia & SCDMTE & $\begin{array}{l}\text { Scientific Centre of } \\
\text { Drug and Medical } \\
\text { Technology } \\
\text { Expertise }\end{array}$ & $\frac{\frac{\text { http://www.pha }}{\text { rm.am/index.ph }}}{\text { p?langid }=2}$ & $\begin{array}{l}\frac{\text { http://apps:wh }}{\text { o.int/medicine }} \\
\frac{\text { sdocs/docume }}{\text { nts/s17079e/s1 }} \\
\frac{7079 \mathrm{e} . \mathrm{pdf}}{}\end{array}$ & 2010 \\
\hline 2 & $\begin{array}{l}\text { Common } \\
\text { wealth of } \\
\text { Independen } \\
\text { t States }\end{array}$ & $\begin{array}{c}\text { Azerbaija } \\
\mathrm{n}\end{array}$ & AECCFM & $\begin{array}{l}\text { Analytical Expertise } \\
\text { Center for Medicines }\end{array}$ & $\frac{\text { http://www.pha }}{\text { rma.az/ }}$ & $\begin{array}{l}\text { Not available } \\
\text { online }\end{array}$ & 2008 \\
\hline 3 & $\begin{array}{l}\text { Common } \\
\text { wealth of } \\
\text { Independen } \\
\text { t States }\end{array}$ & Belarus & & $\begin{array}{l}\text { Ministry of Health- } \\
\text { Dept. of } \\
\text { Pharmaceutical } \\
\text { Inspection }\end{array}$ & $\begin{array}{l}\frac{\text { http://minzdrav. }}{\text { gov.by/en/static }} \\
\text { /about/central_d } \\
\frac{\text { evice/dept Far }}{\text { m/dept farmIns }} \\
\text { pection }\end{array}$ & & \\
\hline 4 & $\begin{array}{l}\text { Common } \\
\text { wealth of } \\
\text { Independen } \\
\text { t States }\end{array}$ & $\begin{array}{c}\text { Kazakhsta } \\
n\end{array}$ & & $\begin{array}{l}\text { National Center for } \\
\text { medicines, medical } \\
\text { devices and medical } \\
\text { equipment expertice }\end{array}$ & $\begin{array}{l}\text { http://www.drai } \\
\text {.kz/?lang=rus }\end{array}$ & & \\
\hline 5 & $\begin{array}{l}\text { Common } \\
\text { wealth of } \\
\text { Independen } \\
\text { t States }\end{array}$ & $\begin{array}{c}\text { Kyrgyz } \\
\text { Republic }\end{array}$ & & & & $\begin{array}{l}\frac{\text { http://www.wh }_{\text {o.int/entity/sel }}}{\text { ection }} \\
\text { medicines/cou } \\
\text { ntry lists/Kyrg } \\
\text { 2009.pdf }\end{array}$ & 2009 \\
\hline 6 & $\begin{array}{l}\text { Common } \\
\text { wealth of } \\
\text { Independen } \\
\text { t States }\end{array}$ & Moldova & AM & $\begin{array}{l}\text { Agentia } \\
\text { Medicamentului }\end{array}$ & $\frac{\frac{\text { http://www.ame }}{\text { d.md/index eng }}}{\underline{. h t m l}}$ & $\begin{array}{l}\frac{\frac{\text { http://www.wh }}{\text { o.int/entity/sel }}}{\frac{\text { ection }}{\text { medicines } / \text { cou }}} \\
\frac{\text { ntry lists/mda }}{\text { 2009.pdf }}\end{array}$ & 2009 \\
\hline 7 & $\begin{array}{l}\text { Common } \\
\text { wealth of } \\
\text { Independen } \\
\text { t States }\end{array}$ & $\begin{array}{c}\text { Russian } \\
\text { Federatio } \\
\text { n }\end{array}$ & & $\begin{array}{l}\text { Federal Service for } \\
\text { Supervision of Health }\end{array}$ & $\frac{\text { http://www.rosz }}{\text { dravnadzor.ru/ }}$ & $\begin{array}{l}\text { Not available } \\
\text { online }\end{array}$ & \\
\hline 8 & $\begin{array}{l}\text { Common } \\
\text { wealth of } \\
\text { Independen } \\
\text { t States }\end{array}$ & Tajikistan & & & & $\begin{array}{l}\frac{\frac{\text { http://www.wh }}{\text { o.int/entity/sel }}}{\text { ection }} \\
\frac{\text { medicines } / \text { cou }}{\text { ntry lists/tjk }} \\
\text { EDL 2009.pdf }\end{array}$ & 2009 \\
\hline 9 & $\begin{array}{l}\text { Common } \\
\text { wealth of } \\
\text { Independen } \\
\text { t States }\end{array}$ & $\begin{array}{l}\text { Ukrain } \\
\mathrm{e}\end{array}$ & & Ministry of Health & $\begin{array}{l}\frac{\text { http://www.pha }}{\text { rma- }} \\
\text { center.kiev.ua/v } \\
\text { iew/farm_news }\end{array}$ & 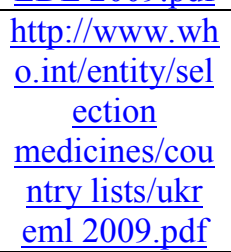 & 2009 \\
\hline
\end{tabular}




\section{LATIN AMERICA \& CARIBBEAN ${ }^{1,2,5,6}$ :}

There are 33 countries in Latin America and the Caribbean today, according to the United Nations. Latin America refers to territories in the Americas where the Spanish or Portuguese languages prevail:
Mexico, most of Central and South America, and in the Caribbean, Cuba, the Dominican Republic, and Puerto Rico. Latin America is, therefore, defined as all those parts of the Americas that were once part of the Spanish and Portuguse.

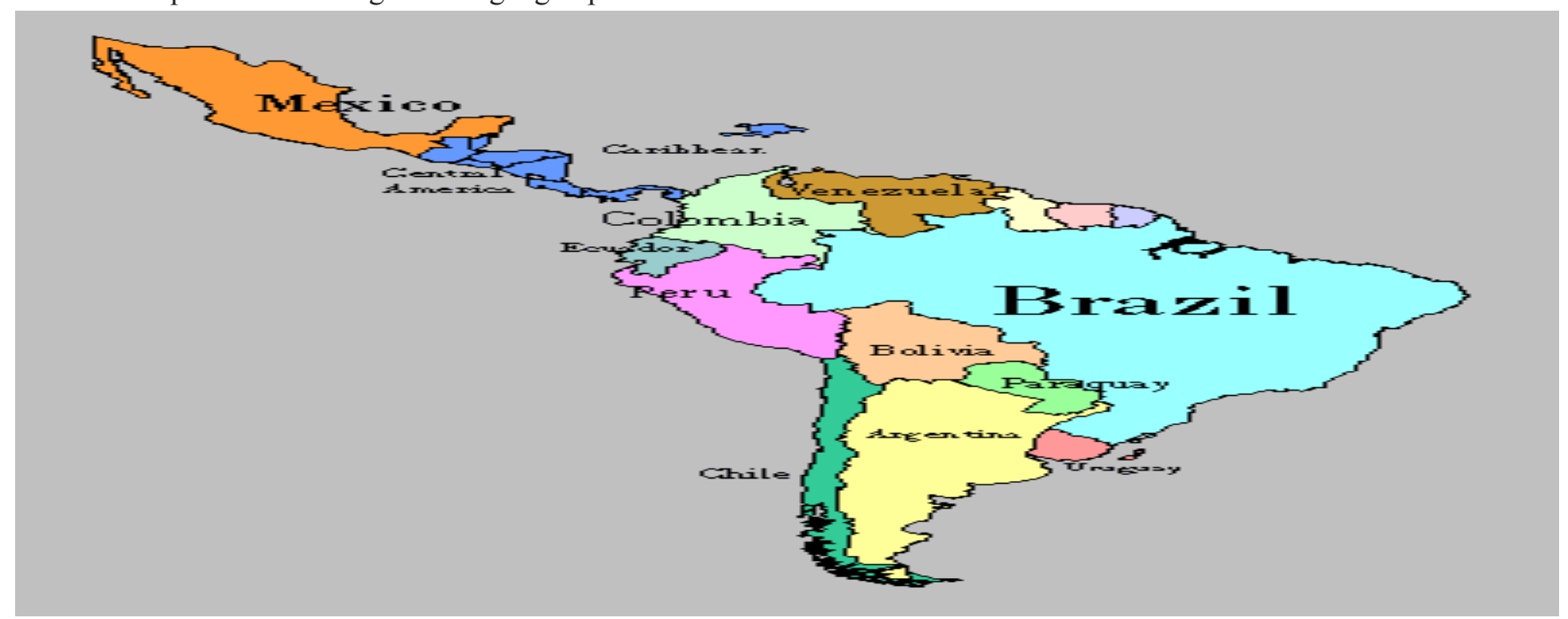

\begin{tabular}{|c|c|c|c|c|c|c|c|}
\hline $\begin{array}{l}\text { Sr. } \\
\text { No }\end{array}$ & Region & Country & $\begin{array}{c}\text { Medicin } \\
\text { es } \\
\text { Agency/ } \\
\text { Authorit } \\
\text { y } \\
\text { Acrony } \\
\text { m }\end{array}$ & $\begin{array}{l}\text { Medicines } \\
\text { Agency / } \\
\text { Authority }\end{array}$ & Website & $\begin{array}{l}\text { Essential Medicines } \\
\text { list }\end{array}$ & $\begin{array}{l}\text { Esse } \\
\text { ntial } \\
\text { Med } \\
\text { icine } \\
\text { s list } \\
\text { year }\end{array}$ \\
\hline 1 & $\begin{array}{c}\text { Latin } \\
\text { America } \\
\text { n \& } \\
\text { Caribbea } \\
\text { n }\end{array}$ & $\begin{array}{l}\text { Antigua } \\
\& \text { Barbuda }\end{array}$ & & & & & \\
\hline 2 & $\begin{array}{l}\text { Latin } \\
\text { America } \\
\mathrm{n} \& \\
\text { Caribbea } \\
\mathrm{n}\end{array}$ & Argentina & ANMAT & $\begin{array}{c}\text { Agencia } \\
\text { Nacional de } \\
\text { Medicamentos, } \\
\text { Alimentos y } \\
\text { Teconologia // } \\
\text { National } \\
\text { Administraton of } \\
\text { Drugs, Food and } \\
\text { Medical } \\
\text { Technologies }\end{array}$ & $\frac{\text { http://www.anmat.gov.ar }}{I}$ & $\begin{array}{c}\frac{\text { http://www.who.int/en }}{\text { tity/selection }} \\
\frac{\begin{array}{l}\text { medicines/country } \\
\text { lists/arg MEML }\end{array}}{\underline{2005 . \mathrm{pdf}}}\end{array}$ & 2005 \\
\hline 3 & $\begin{array}{l}\text { Latin } \\
\text { America }\end{array}$ & Bahama & & Bahamas & $\frac{\text { http://www.pharmacyco }}{\text { uncil.net/ }}$ & & \\
\hline
\end{tabular}




\begin{tabular}{|c|c|c|c|c|c|c|c|}
\hline & $\begin{array}{l}\mathrm{n} \& \\
\text { Caribbea } \\
\mathrm{n}\end{array}$ & $\mathrm{s}$ & & $\begin{array}{l}\text { Pharmacy } \\
\text { Council }\end{array}$ & & & \\
\hline 4 & $\begin{array}{l}\text { Latin } \\
\text { America } \\
\mathrm{n} \& \\
\text { Caribbea } \\
\mathrm{n}\end{array}$ & Barbados & $\mathrm{MOH}$ & Ministry of Health & $\frac{\text { http://www.health.gov.b }}{\underline{\mathrm{b}}}$ & $\frac{\underline{\text { http://apps.who.int } / \mathrm{me}}}{\underline{\text { dicinedocs/en/m/abstra }}} \frac{\text { ct/Js18839en/ }}{}$ & $\begin{array}{l}2011 \\
- \\
2012\end{array}$ \\
\hline 5 & $\begin{array}{l}\text { Latin } \\
\text { America } \\
\mathrm{n} \& \\
\text { Caribbea } \\
\mathrm{n}\end{array}$ & Belize & $\mathrm{MOH}$ & Ministry of Health & $\frac{\text { http://health.gov.bz/ww }}{\text { w/units/pharmacy }}$ & $\begin{array}{l}\text { http://www.who.int/en } \\
\begin{array}{c}\text { tity/selection } \\
\text { medicines/country }\end{array} \\
\frac{\text { lists/blz formulary }}{\underline{200911 . p d f}}\end{array}$ & $\begin{array}{c}2009 \\
- \\
2011\end{array}$ \\
\hline 6 & $\begin{array}{l}\text { Latin } \\
\text { America } \\
\mathrm{n} \& \\
\text { Caribbea } \\
\mathrm{n}\end{array}$ & $\begin{array}{l}\text { Bermud } \\
\quad \text { a }\end{array}$ & BPA & $\begin{array}{c}\text { Bermuda } \\
\text { Pharmaceutical } \\
\text { Association }\end{array}$ & $\frac{\text { http://www.bpa.bm/coun }}{\underline{\text { cil.htm }}}$ & & \\
\hline 7 & $\begin{array}{l}\text { Latin } \\
\text { America } \\
\mathrm{n} \& \\
\text { Caribbea } \\
\mathrm{n}\end{array}$ & Bolivia & $\begin{array}{l}\text { UNIME } \\
\text { D/MSD3 } \\
2865\end{array}$ & $\begin{array}{c}\text { Unidad de } \\
\text { Medicamentos } \\
\text { y Tecnología en } \\
\text { Salud (MSD) }\end{array}$ & $\frac{\text { http://unimed.sns.gob.bo }}{\text { /unimed.index.htm }}$ & $\begin{array}{l}\frac{\frac{\mathrm{http}: / / \text { apps.who.int/me }}{\text { dicinedocs/documents/ }}}{\frac{\text { s18843es/s18843es.pd }}{\underline{f}}} \\
\end{array}$ & \\
\hline 8 & $\begin{array}{l}\text { Latin } \\
\text { America } \\
\mathrm{n} \& \\
\text { Caribbea } \\
\mathrm{n}\end{array}$ & Brazil & ANVISA & $\begin{array}{l}\text { National } \\
\text { Health } \\
\text { Surveillan } \\
\text { ce Agency }\end{array}$ & $\begin{array}{l}\text { http://portal.anvisa.gov.b } \\
\text { r/wps/portal/anvisa/home }\end{array}$ & $\begin{array}{c}\text { http://www.who.int/en } \\
\begin{array}{c}\text { tity/selection } \\
\text { medicines/country }\end{array} \\
\underline{\text { lists/Brazil }} \\
\text { rename2010.pdf }\end{array}$ & \\
\hline 9 & $\begin{array}{l}\text { Latin } \\
\text { America } \\
\mathrm{n} \& \\
\text { Caribbea } \\
\mathrm{n}\end{array}$ & $\begin{array}{l}\text { British } \\
\text { Virgin } \\
\text { Islands }\end{array}$ & & & & & \\
\hline 10 & $\begin{array}{l}\text { Latin } \\
\text { America } \\
\mathrm{n} \& \\
\text { Caribbea } \\
\mathrm{n}\end{array}$ & $\begin{array}{l}\text { Cayman } \\
\text { Islands }\end{array}$ & & & & & \\
\hline 11 & $\begin{array}{l}\text { Latin } \\
\text { America } \\
\mathrm{n} \& \\
\text { Caribbea } \\
\mathrm{n}\end{array}$ & Colombia & INVIMA & $\begin{array}{c}\text { Instituto } \\
\text { Nacional de } \\
\text { Vigilancia de } \\
\text { Medicamento }\end{array}$ & $\frac{\mathrm{http}: / / \text { www.invima.gov.c }}{\underline{\mathrm{o} /}}$ & $\begin{array}{l}\frac{\frac{\mathrm{http}: / / \text { apps.who.int/me }}{\text { dicinedocs/documents// }}}{\frac{\text { s18382es/s18382es.pd }}{\mathrm{f}}} \\
\end{array}$ & \\
\hline
\end{tabular}




\begin{tabular}{|c|c|c|c|c|c|c|}
\hline & & & & $\begin{array}{l}\text { s y } \\
\text { Alimentos }\end{array}$ & & \\
\hline 12 & $\begin{array}{l}\text { Latin } \\
\text { America } \\
\mathrm{n} \& \\
\text { Caribbea } \\
\mathrm{n}\end{array}$ & CostaRica & MINSA & $\begin{array}{c}\text { Centro } \\
\text { Nacional de } \\
\text { Farmacovigil } \\
\text { ancia/MINS } \\
\text { A }\end{array}$ & $\begin{array}{l}\frac{\text { http://www.minisrteriode }}{\text { salud.go.cr/index.php/far }} \\
\frac{\text { macovigilancia-alerta- }}{\text { seguridad-ms/cat }} \\
\text { view/191-regulacion-de- }\end{array}$ & \\
\hline 13 & $\begin{array}{l}\text { Latin } \\
\text { America } \\
\mathrm{n} \& \\
\text { Caribbea } \\
\mathrm{n}\end{array}$ & Cuba & $\begin{array}{l}\text { CECME } \\
\text { D }\end{array}$ & $\begin{array}{c}\text { Center for } \\
\text { State Control } \\
\text { of Drug } \\
\text { Qualtiy Cuba }\end{array}$ & http://www.cemed.sld.cu & \\
\hline 14 & $\begin{array}{l}\text { Latin } \\
\text { America } \\
\mathrm{n} \& \\
\text { Caribbea } \\
\mathrm{n}\end{array}$ & $\begin{array}{l}\text { Curacao } \\
\text { (former } \\
\text { Netherland } \\
\text { Antilles) }\end{array}$ & & & & \\
\hline 15 & $\begin{array}{l}\text { Latin } \\
\text { America } \\
\mathrm{n} \& \\
\text { Caribbea } \\
\mathrm{n} \\
\end{array}$ & Dominica & & & & \\
\hline 16 & $\begin{array}{l}\text { Latin } \\
\text { America } \\
\mathrm{n} \& \\
\text { Caribbea } \\
\mathrm{n}\end{array}$ & $\begin{array}{l}\text { Dominican } \\
\text { Republic }\end{array}$ & $\begin{array}{c}\text { DGDF/ } \\
\text { MSP }\end{array}$ & $\begin{array}{c}\text { Direccion } \\
\text { General de } \\
\text { Drogas y } \\
\text { Farmacias del } \\
\text { Ministerio de } \\
\text { Salud Pública }\end{array}$ & $\frac{\text { http://www.drogasyfarm }}{\underline{\text { acias.gov.do }}}$ & $\begin{array}{l}\frac{\underline{\text { http://apps.who.int } / \mathrm{me}}}{\text { dicinedocs/documents/ }} \\
\frac{\text { s18241es/s18241es.pd }}{\underline{f}}\end{array}$ \\
\hline 17 & $\begin{array}{l}\text { Latin } \\
\text { America } \\
\mathrm{n} \& \\
\text { Caribbea } \\
\mathrm{n}\end{array}$ & Ecuador & & $\begin{array}{l}\text { Ministerio de } \\
\text { Salud Pública }\end{array}$ & www.salud.gob.ec/ & 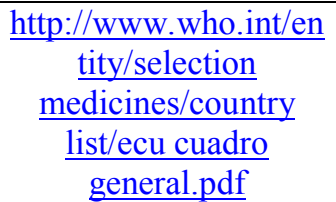 \\
\hline 18 & $\begin{array}{l}\text { Latin } \\
\text { America } \\
\mathrm{n} \& \\
\text { Caribbea } \\
\mathrm{n}\end{array}$ & ElSalvador & CSSP & $\begin{array}{l}\text { Consejo Superior } \\
\text { de Salud Pública }\end{array}$ & http://www.cssp.gob.sv/ & $\begin{array}{c}\frac{\mathrm{http} / / \text { asp.mspas.gob.s }}{\mathrm{v} / \mathrm{regulacion} / \mathrm{pdf} / \text { listad }} \\
\frac{\text { os/Listado Oficial de }}{\text { medicamentos } 10 \mathrm{a}} \\
\text { version Adendas } 2011 \\
\text { Inclusiones } \\
\end{array}$ \\
\hline 19 & $\begin{array}{l}\text { Latin } \\
\text { America } \\
\mathrm{n} \& \\
\text { Caribbea } \\
\mathrm{n} \\
\end{array}$ & Grenada & & $\begin{array}{l}\text { Ministry } \\
\text { of Health: } \\
\text { Pharmacy } \\
\text { Council }\end{array}$ & & \\
\hline 20 & $\begin{array}{l}\text { Latin } \\
\text { America } \\
\mathrm{n} \& \\
\text { Caribbea }\end{array}$ & Guatemala & MSPAS & $\begin{array}{l}\text { Minsiterio } \\
\text { de Salud } \\
\text { Publica y } \\
\text { Asistencia }\end{array}$ & $\begin{array}{c}\text { http://portal.mspas.gob.g } \\
\text { t/regulation_y_control_d } \\
\text { e_productos } \\
\text { farmaceuticos y }\end{array}$ & \\
\hline
\end{tabular}




\begin{tabular}{|c|c|c|c|c|c|c|c|}
\hline & $\mathrm{n}$ & & & 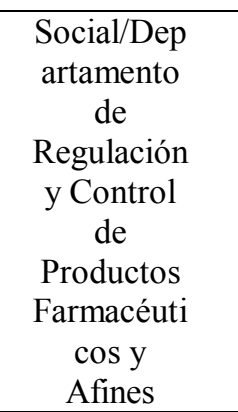 & afines.html & & \\
\hline 21 & $\begin{array}{l}\text { Latin } \\
\text { America } \\
\mathrm{n} \& \\
\text { Caribbea } \\
\mathrm{n}\end{array}$ & Guyana & & & $\begin{array}{l}\text { http://www.health.gov.g } \\
\text { v/prgadm food drugs.php }\end{array}$ & $\begin{array}{l}\frac{\text { http://www.who.int/en }}{\begin{array}{c}\text { tity/selection } \\
\text { medicines/country }\end{array}} \\
\frac{\text { lists/GUYANA EDL }}{2009 \text { 10.pdf }}\end{array}$ & $\begin{array}{c}2009 \\
- \\
2011\end{array}$ \\
\hline 22 & $\begin{array}{l}\text { Latin } \\
\text { America } \\
\mathrm{n} \& \\
\text { Caribbea } \\
\mathrm{n}\end{array}$ & Haiti & & & & $\begin{array}{c}\frac{\text { http://www.who.int/en }}{\text { tity/selection }} \\
\frac{\text { medicines/country }}{\text { lists/Haiti liste } 2003} \\
\text { 2008.pdf }\end{array}$ & $\begin{array}{c}2003 \\
- \\
2008\end{array}$ \\
\hline 23 & $\begin{array}{l}\text { Latin } \\
\text { America } \\
\mathrm{n} \& \\
\text { Caribbea } \\
\mathrm{n}\end{array}$ & Honduras & DGRS & $\begin{array}{l}\text { Direccion } \\
\text { General de } \\
\text { Regulacion } \\
\text { Sanitaria }\end{array}$ & http://www.dgrs.gob.hn/ & $\begin{array}{l}\text { http://apps.who.int/me } \\
\text { dicinedocs/documents/ } \\
\underline{\text { s18372es/s18372es.pd }}\end{array}$ & $\begin{array}{c}2009 \\
- \\
2011\end{array}$ \\
\hline 24 & $\begin{array}{l}\text { Latin } \\
\text { America } \\
\mathrm{n} \& \\
\text { Caribbea } \\
\mathrm{n}\end{array}$ & Jamaica & PCOJ & $\begin{array}{l}\text { Pharmacy } \\
\text { Council of } \\
\text { Jamaica }\end{array}$ & http://www.pcoj.org/ & $\begin{array}{c}\frac{\text { http://www.who.int/en }}{\text { tity/selection }} \\
\frac{\text { medicines/country }}{\text { lists/Jamaica }} \\
\underline{\text { 2008.PDF }}\end{array}$ & 2008 \\
\hline 25 & $\begin{array}{l}\text { Latin } \\
\text { America } \\
\mathrm{n} \& \\
\text { Caribbea } \\
\mathrm{n}\end{array}$ & Monsterrat & & & & & \\
\hline 26 & $\begin{array}{l}\text { Latin } \\
\text { America } \\
\mathrm{n} \& \\
\text { Caribbea } \\
\mathrm{n}\end{array}$ & Nicaragua & MINSA & $\begin{array}{l}\text { Ministerio } \\
\text { de Salud - } \\
\text { Direccion de } \\
\text { Farmacias }\end{array}$ & www.minsa.gob.ni & $\begin{array}{c}\frac{\underline{\text { http://www.minsa.gob. }}}{\text { ni/indexphp?option }=\mathrm{c}} \\
\underline{\text { om }} \\
\text { remository\&Itemid }=5 \\
\text { 2\&func=fileinfo\&id }=6 \\
847\end{array}$ & 2011 \\
\hline 27 & $\begin{array}{l}\text { Latin } \\
\text { America } \\
\mathrm{n} \& \\
\text { Caribbea } \\
\mathrm{n}\end{array}$ & Panama & MINSA & $\begin{array}{l}\text { Ministerio de } \\
\text { Salud }\end{array}$ & $\frac{\text { http://www.minsa.gob.pa }}{I}$ & & \\
\hline 28 & $\begin{array}{l}\text { Latin } \\
\text { America } \\
\mathrm{n} \& \\
\text { Caribbea }\end{array}$ & Paraguay & MSPBS & $\begin{array}{l}\text { Ministerio de } \\
\text { Salud } \\
\text { Pública y }\end{array}$ & $\frac{\text { http://www.mspbs.gov.p }}{\mathrm{y} /}$ & $\begin{array}{c}\frac{\text { http://www.who.int.en }}{\text { tity/selection }} \\
\text { medicines/country } \\
\underline{\text { lists/pry 2009.pdf }}\end{array}$ & 2009 \\
\hline
\end{tabular}




\begin{tabular}{|c|c|c|c|c|c|c|c|}
\hline & $\mathrm{n}$ & & & $\begin{array}{l}\text { Bienestar } \\
\text { Social }\end{array}$ & & & \\
\hline 29 & $\begin{array}{l}\text { Latin } \\
\text { America } \\
\mathrm{n} \& \\
\text { Caribbea } \\
\mathrm{n}\end{array}$ & Peru & $\begin{array}{l}\text { DIGIME } \\
\text { D- } \\
\text { MINSA }\end{array}$ & $\begin{array}{l}\text { Dirección } \\
\text { General } \\
\text { de } \\
\text { Medicame } \\
\text { ntos }\end{array}$ & $\frac{\text { http://www.digemed.min }}{\underline{\text { sa.gob.pe/ }}}$ & $\begin{array}{l}\frac{\text { http://www.who.int.en }}{\text { tity/selection }} \\
\underline{\text { medicines/country }} \\
\underline{\text { lists/PER 2010.pdf }}\end{array}$ & 2010 \\
\hline 30 & $\begin{array}{l}\text { Latin } \\
\text { America } \\
\mathrm{n} \& \\
\text { Caribbea } \\
\mathrm{n} \\
\end{array}$ & $\begin{array}{c}\text { SaintKittsa } \\
\text { ndNevis }\end{array}$ & & $\begin{array}{l}\text { Ministry of } \\
\text { Health }\end{array}$ & $\frac{\underline{\text { http://www.gov.kn/direct }}}{\text { ory?q=moh }}$ & & \\
\hline 31 & $\begin{array}{l}\text { Latin } \\
\text { America } \\
\mathrm{n} \& \\
\text { Caribbea } \\
\mathrm{n}\end{array}$ & SaintLucia & & $\begin{array}{l}\text { Ministry of } \\
\text { Health }\end{array}$ & $\frac{\text { http://www.stulucia.gov. }}{\underline{\text { Ic/ }}}$ & & \\
\hline 32 & $\begin{array}{l}\text { Latin } \\
\text { America } \\
\mathrm{n} \& \\
\text { Caribbea } \\
\mathrm{n}\end{array}$ & $\begin{array}{l}\text { SaintVince } \\
\text { ntandthe } \\
\text { Grenadines }\end{array}$ & & $\begin{array}{c}\text { St. Vincent \& } \\
\text { the } \\
\text { Grenadines } \\
\text { Pharmacy } \\
\text { Council }\end{array}$ & & $\begin{array}{l}\frac{\frac{\text { http://apps.who.int..me }}{\text { dicinedocs/documents/ }}}{\underline{\text { s18854en/s18854en.pd }}} \\
\underline{\mathrm{f}}\end{array}$ & 2010 \\
\hline 33 & $\begin{array}{l}\text { Latin } \\
\text { America } \\
\mathrm{n} \& \\
\text { Caribbea } \\
\mathrm{n}\end{array}$ & Suriname & & $\begin{array}{l}\text { Pharmaceut } \\
\quad \text { ical } \\
\text { Inspectorate } \\
\text { and the } \\
\text { Registration } \\
\text { committee }\end{array}$ & & $\begin{array}{l}\frac{\text { http://www.who.int.en }}{\text { tity/selection }} \\
\text { medicines/countrylists } \\
\text { /Suriname 2004.pdf }\end{array}$ & 2004 \\
\hline 34 & $\begin{array}{l}\text { Latin } \\
\text { America } \\
\mathrm{n} \& \\
\text { Caribbea } \\
\mathrm{n} \\
\end{array}$ & $\begin{array}{c}\text { Trinidad\& } \\
\text { Tobago }\end{array}$ & $\begin{array}{l}\mathrm{CFDD} / \\
\mathrm{MOH}\end{array}$ & $\begin{array}{l}\text { Chemistry Food } \\
\text { and Drugs } \\
\text { Division/Minist } \\
\text { ry of Health }\end{array}$ & $\begin{array}{c}\frac{\text { http://www.health.gov.tt/ }}{\text { sitepages/default.aspx?id }} \\
=93\end{array}$ & $\begin{array}{l}\frac{\text { http://www.who.int.en }}{\text { tity/selection }} \\
\text { medicines/countrylists } \\
\text { /Tobago 2010.pdf }\end{array}$ & 2010 \\
\hline 35 & $\begin{array}{l}\text { Latin } \\
\text { America } \\
\mathrm{n} \& \\
\text { Caribbea } \\
\mathrm{n} \\
\end{array}$ & $\begin{array}{l}\text { Turks \& } \\
\text { Caicos } \\
\text { Islands }\end{array}$ & & & & & \\
\hline 36 & $\begin{array}{l}\text { Latin } \\
\text { America } \\
\mathrm{n} \& \\
\text { Caribbea } \\
\mathrm{n}\end{array}$ & Uruguay & MSP & $\begin{array}{l}\text { Ministerio de } \\
\text { Salud } \\
\text { Publica. } \\
\text { Direccion } \\
\text { General de la } \\
\text { Salud }\end{array}$ & $\begin{array}{l}\text { http://www.msp.gub.uy/ } \\
\text { egorias_176_1.html }\end{array}$ & $\begin{array}{l}\frac{\underline{\text { http://apps.who.int/me }}}{\underline{\text { dicined }}} \\
\underline{\text { ocs/documents/s1871 }} \\
\underline{\underline{\text { es } / \mathrm{s} 187}} \\
\underline{11 \mathrm{es} . \mathrm{pdf}}\end{array}$ & 2011 \\
\hline 37 & Latin & Venezuela & INHRR & & & http://www.who.int/ent & 2004 \\
\hline
\end{tabular}




\begin{tabular}{|c|c|c|c|c|}
\hline $\begin{array}{l}\text { America } \\
\mathrm{n} \& \\
\text { Caribbea } \\
\mathrm{n}\end{array}$ & /MPPS & $\begin{array}{c}\text { Instituto } \\
\text { Nacional de } \\
\text { Higiene "Rafael } \\
\text { Rangel"/Ministe } \\
\text { rio de Salud }\end{array}$ & $\begin{array}{c}\text { http://www.inhrr.gob.v } \\
\text { e/ }\end{array}$ & $\begin{array}{l}\frac{\frac{\text { ity/sele }}{\text { ction_medicines/count }}}{\text { ry_lists/ }} \\
\text { ven_2004_medicamen } \\
\text { tos_esen ciales.pdf }\end{array}$ \\
\hline
\end{tabular}

\section{OECD-Eurostat:}

On 14 December 1960, 20 countries originally signed the Convention on the Organisation for Economic
Co-operation and Development. Since then, 15 countries have become members of the Organisation.

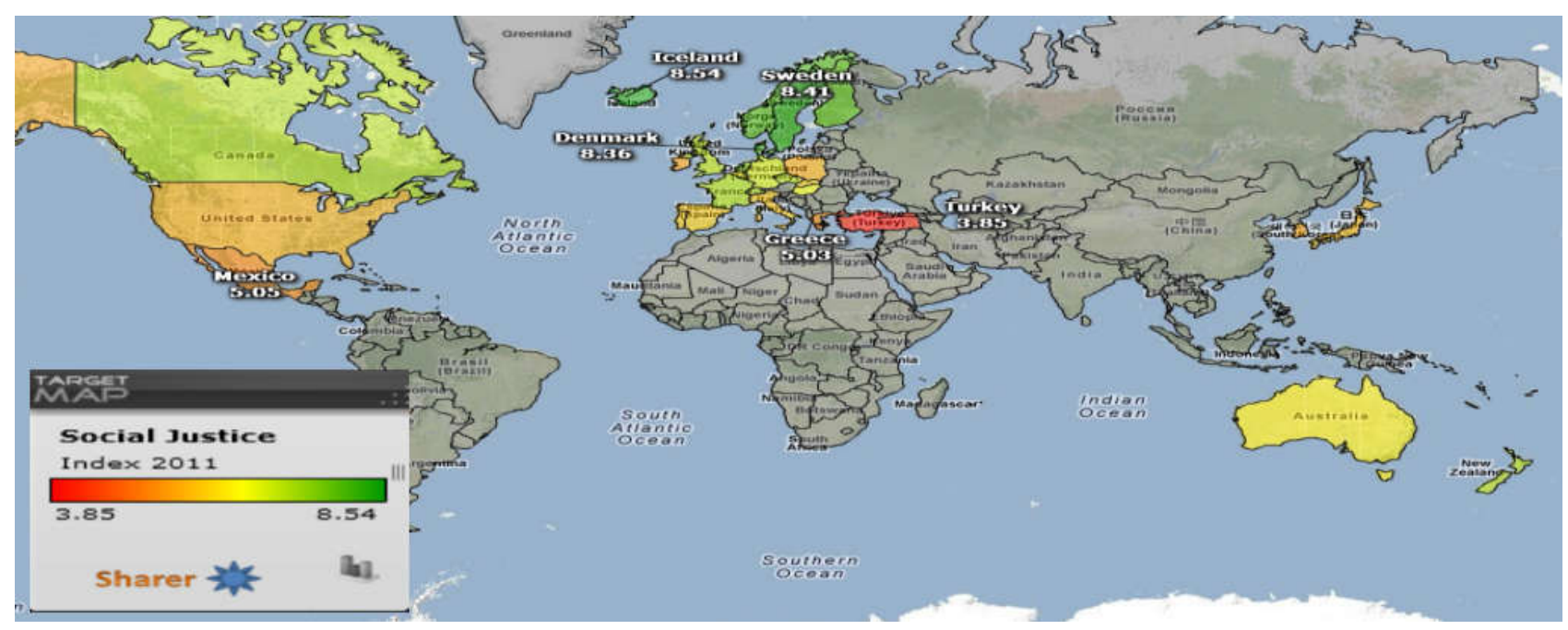

\begin{tabular}{|c|c|c|c|c|c|c|c|}
\hline $\begin{array}{l}\text { Sr. } \\
\text { No }\end{array}$ & Region & Country & $\begin{array}{l}\text { Medicines } \\
\text { Agency/ } \\
\text { Authority } \\
\text { Acronym }\end{array}$ & $\begin{array}{l}\text { Medicines Agency / } \\
\text { Authority }\end{array}$ & Website & $\begin{array}{c}\text { Essential } \\
\text { Medicines } \\
\quad \text { list }\end{array}$ & $\begin{array}{l}\text { Essent } \\
\text { ial } \\
\text { Medici } \\
\text { nes list } \\
\text { year }\end{array}$ \\
\hline 1 & $\begin{array}{l}\text { OECD - } \\
\text { Eurostat }\end{array}$ & Albania & QKKB & $\begin{array}{l}\text { National Drug Control } \\
\text { Center }\end{array}$ & $\frac{\underline{\text { http://www }}}{\frac{\text { qkkb.gov.a }}{1 /}}$ & & \\
\hline 2 & $\begin{array}{l}\text { OECD- } \\
\text { Eurostat }\end{array}$ & Australia & TGA & $\begin{array}{c}\text { Australia: Therapeutic } \\
\text { Goods } \\
\text { Administration }\end{array}$ & $\begin{array}{l}\text { http://www } \\
\text {.tga.gov.au } \\
/ /\end{array}$ & & \\
\hline 3 & $\begin{array}{l}\text { OECD- } \\
\text { Eurostat }\end{array}$ & Austria & $\mathrm{MOH}$ & $\begin{array}{l}\text { Federal Ministry of } \\
\text { Health }\end{array}$ & $\frac{\text { http://www }}{\text { bmgfj.gv.a }}$ & & \\
\hline 4 & $\begin{array}{l}\text { OECD- } \\
\text { Eurostat }\end{array}$ & Belgium & FAMHP & $\begin{array}{l}\text { Federal Agency for } \\
\text { Medicines and }\end{array}$ & http://www.f & & \\
\hline
\end{tabular}




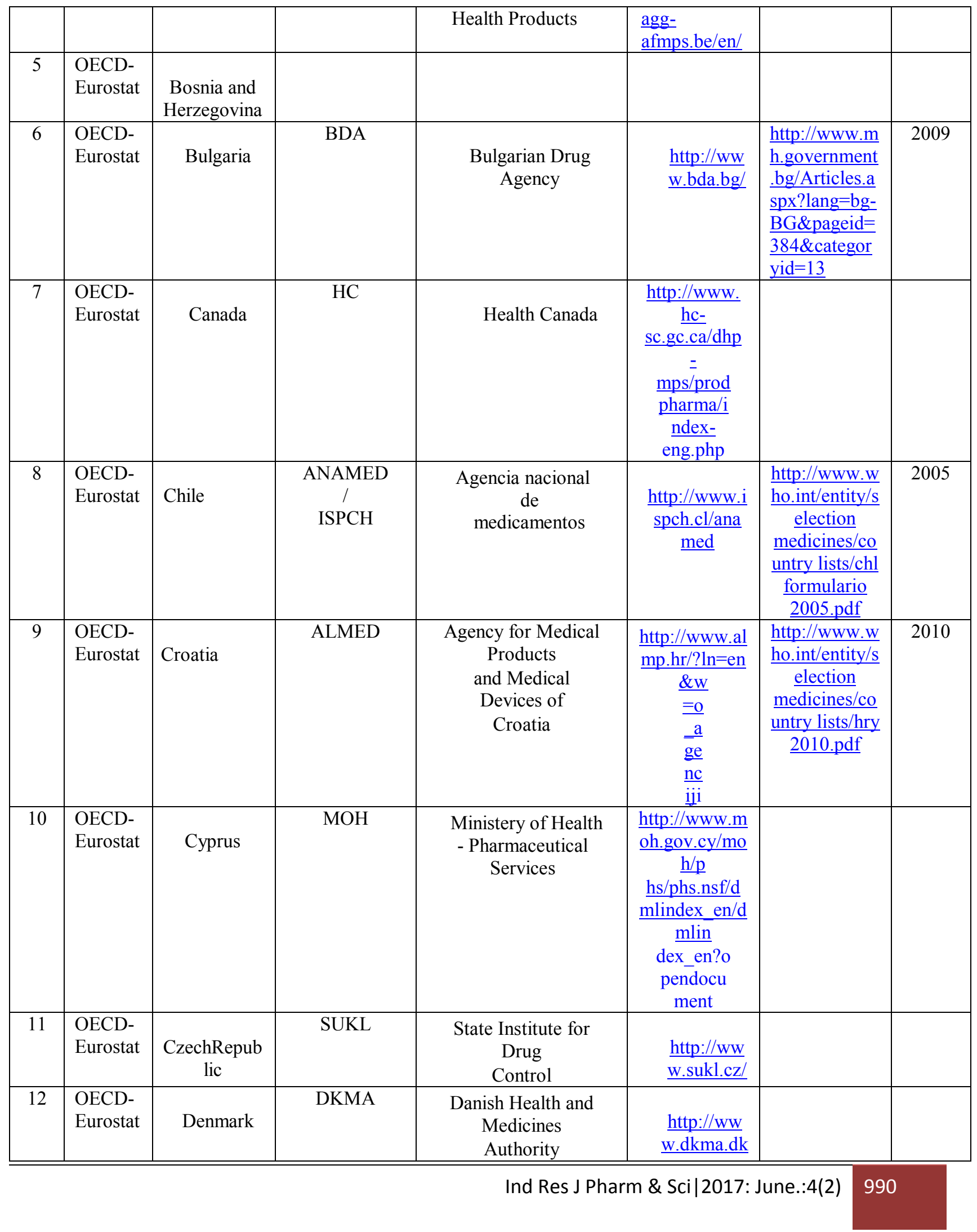




\begin{tabular}{|c|c|c|c|c|c|c|c|}
\hline & & & & & 1 & & \\
\hline 13 & $\begin{array}{l}\text { OECD- } \\
\text { Eurostat }\end{array}$ & Estonia & SAM & $\begin{array}{l}\text { State Agency of } \\
\text { Medicines }\end{array}$ & $\frac{\text { http://ww }}{\text { w.sam.ee/ }}$ & & \\
\hline 14 & $\begin{array}{l}\text { OECD- } \\
\text { Eurostat }\end{array}$ & Finland & FIMEA & $\begin{array}{c}\text { Finnish Medicines } \\
\text { Agency }\end{array}$ & $\frac{\frac{h t t p: / / w w}{\text { w.fimea.fi }}}{!}$ & & \\
\hline 15 & $\begin{array}{l}\text { OECD- } \\
\text { Eurostat }\end{array}$ & France & ANSM & $\begin{array}{c}\text { National Agency for } \\
\text { the } \\
\text { Safety of Medicine } \\
\text { and } \\
\text { Health Products }\end{array}$ & $\frac{\text { www.ans }}{\text { m.sante.fr }}$ & & \\
\hline 16 & $\begin{array}{l}\text { OECD- } \\
\text { Eurostat }\end{array}$ & Germany & BfArM & $\begin{array}{l}\text { Federal Institute for } \\
\text { Drugs and Medical } \\
\text { Services and }\end{array}$ & $\frac{\frac{\mathrm{http}: / / \mathrm{ww}}{\mathrm{w} \cdot \mathrm{bfarm} \cdot \mathrm{d}}}{\mathrm{e} /}$ & & \\
\hline 17 & $\begin{array}{l}\text { OECD- } \\
\text { Eurostat }\end{array}$ & Greece & EOF & $\begin{array}{l}\text { National } \\
\text { Organization for } \\
\text { Medicines }\end{array}$ & 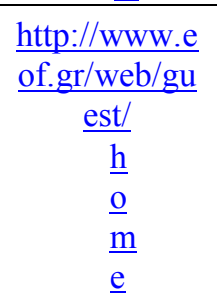 & & \\
\hline 18 & $\begin{array}{l}\text { OECD- } \\
\text { Eurostat }\end{array}$ & Hungary & & $\begin{array}{c}\text { Directorate General } \\
\text { of } \\
\text { National Institute } \\
\text { of } \\
\text { Pharmacy }\end{array}$ & $\frac{\frac{\text { http://www.o }}{\text { gyi.hu/main }}}{\frac{\text { page }}{/}}$ & & \\
\hline 19 & $\begin{array}{l}\text { OECD- } \\
\text { Eurostat }\end{array}$ & Iceland & IMCA & $\begin{array}{c}\text { Icelandic Medicines } \\
\text { Agency }\end{array}$ & $\underline{\text { http://ww }}$ & & \\
\hline 20 & $\begin{array}{l}\text { OECD- } \\
\text { Eurostat }\end{array}$ & Ireland & IMB & $\begin{array}{l}\text { Irish Medicines } \\
\text { Board }\end{array}$ & $\underline{\text { http://ww }}$ & & \\
\hline 21 & $\begin{array}{l}\text { OECD- } \\
\text { Eurostat }\end{array}$ & Israel & $\mathrm{MOH}$ & Ministry of Health & $\begin{array}{l}\frac{\text { http://www.h }}{\text { ealth.gov.il/E }} \\
\text { nglis } \\
\frac{\text { h/MinistryUni }}{\frac{\text { ts/Pages/Units }}{\underline{\text { List }}}} \\
\text {.aspx }\end{array}$ & & \\
\hline 22 & $\begin{array}{l}\text { OECD- } \\
\text { Eurostat }\end{array}$ & Italy & AIFA & $\begin{array}{c}\text { Italian Medicines } \\
\text { Agency }\end{array}$ & $\frac{\frac{\text { http://www.a }}{\text { genziafarmac }}}{\underline{\text { o.it/ }}}$ & & \\
\hline 23 & $\begin{array}{l}\text { OECD- } \\
\text { Eurostat }\end{array}$ & Japan & PMDA & $\begin{array}{c}\text { Pharmaceuticals and } \\
\text { Medical } \\
\text { Devices Agency, }\end{array}$ & $\frac{\underline{\text { http://www.p }}}{\underline{\text { mda.go.jp/eng }}} \underline{\underline{\text { lish }}}$ & & \\
\hline
\end{tabular}




\begin{tabular}{|c|c|c|c|c|c|c|c|}
\hline & & & & Japan & $\frac{\text { ndex.htm }}{1}$ & & \\
\hline 24 & $\begin{array}{l}\text { OECD- } \\
\text { Eurostat }\end{array}$ & Korea, Rep. & KFDA & $\begin{array}{l}\text { Korea Food \& } \\
\text { Drug } \\
\text { Administration }\end{array}$ & $\frac{\frac{\mathrm{http}: / / \mathrm{www} .}{\mathrm{kfda} \cdot \mathrm{go} \cdot \mathrm{kr} / \mathrm{e}}}{\mathrm{ng} /}$ & & \\
\hline 25 & $\begin{array}{l}\text { OECD- } \\
\text { Eurostat }\end{array}$ & Latvia & & $\begin{array}{l}\text { State Agency of } \\
\text { Medicines }\end{array}$ & $\frac{\text { www.zva.gov.1 }}{\underline{\mathrm{v}}}$ & & \\
\hline 26 & $\begin{array}{l}\text { OECD- } \\
\text { Eurostat }\end{array}$ & Lithuania & & $\begin{array}{l}\text { State Medicines } \\
\text { Control } \\
\text { Agency }\end{array}$ & $\frac{\text { http://www.v }}{\underline{\text { vkt.lt/ }}}$ & & \\
\hline 27 & $\begin{array}{l}\text { OECD- } \\
\text { Eurostat }\end{array}$ & Luxembourg & $\mathrm{MOH}$ & Ministry of Health & $\frac{\text { www.ms.etat }}{\underline{. l u}}$ & & \\
\hline 28 & $\begin{array}{l}\text { OECD- } \\
\text { Eurostat }\end{array}$ & $\begin{array}{l}\text { Macedonia } \\
\text { (Former } \\
\text { Yugoslav } \\
\text { Republic) }\end{array}$ & & & & $\begin{array}{l}\frac{\underline{\text { http://www.w }}}{\text { ho.int/entity/s }} \\
\frac{\text { election }}{\text { medicines/co }} \\
\underline{\text { untry }} \\
\underline{\text { lists/mkd }} \\
\underline{2010 . \mathrm{pdf}}\end{array}$ & 2010 \\
\hline 29 & $\begin{array}{l}\text { OECD- } \\
\text { Eurostat }\end{array}$ & Malta & & Medicines Authority & $\begin{array}{l}\frac{\text { http://www.m }}{\text { edicinesautho }} \\
\frac{\text { rity }}{\text { gov.mt }} \\
\text { ! }\end{array}$ & $\begin{array}{l}\frac{\text { http://www.w }}{\text { ho.int/entity/s }} \\
\text { election } \\
\text { medicines/co } \\
\frac{\text { untry lists/mlt }}{\text { Medicinelist }} \\
\underline{\text { April08.pdf }}\end{array}$ & 2008 \\
\hline 30 & $\begin{array}{l}\text { OECD- } \\
\text { Eurostat }\end{array}$ & Mexico & COFEPRIS & $\begin{array}{c}\text { Comisión Federal } \\
\text { para la } \\
\text { Protección contra } \\
\text { Riesgos } \\
\text { Sanitarios / Secretaria } \\
\text { de }\end{array}$ & $\frac{\frac{\text { http://www.c }}{\text { ofepris.gob. }}}{\underline{\mathrm{mx} /}}$ & $\begin{array}{l}\text { http://www.cs } \\
\text { g.salud.gob.m } \\
\text { x/descargas/p } \\
\text { dfs/cuadro } \\
\text { basico/medic } \\
\text { amentos/medi } \\
\text { camentos e }\end{array}$ & 2010 \\
\hline 31 & $\begin{array}{l}\text { OECD- } \\
\text { Eurostat }\end{array}$ & Montenegro & & $\begin{array}{c}\text { Agency for Medical } \\
\text { Products and } \\
\text { Medical Devices }\end{array}$ & $\begin{array}{l}\text { http://sntcg } \\
. \text { com/ulms/ }\end{array}$ & $\begin{array}{l}\frac{\text { http://www.w }}{\text { ho.int/entity/s }} \\
\frac{\text { election }}{\text { medicines/co }} \\
\frac{\text { untry }}{\text { lists/Montene }} \\
\text { gro2011.pdf }\end{array}$ & 2011 \\
\hline 32 & $\begin{array}{l}\text { OECD- } \\
\text { Eurostat }\end{array}$ & $\begin{array}{l}\text { Netherlands, } \\
\text { The }\end{array}$ & MEB & $\begin{array}{c}\text { Medicines Evaluation } \\
\text { Board }\end{array}$ & $\begin{array}{l}\frac{\text { http://www.c }}{\underline{\text { bg- }}} \\
\frac{\text { meb.hl/cbg/e }}{\underline{\mathrm{n} /}}\end{array}$ & & \\
\hline 33 & $\begin{array}{l}\text { OECD- } \\
\text { Eurostat }\end{array}$ & NewZealand & MEDSAFE & $\begin{array}{l}\text { Medicines and } \\
\text { Medical } \\
\text { Devices Safety } \\
\end{array}$ & $\begin{array}{l}\text { http://www. } \\
\text { medsafe.gov }\end{array}$ & & \\
\hline
\end{tabular}




\begin{tabular}{|c|c|c|c|c|c|c|c|}
\hline & & & & Authority & t.nz/ & & \\
\hline 34 & $\begin{array}{l}\text { OECD- } \\
\text { Eurostat }\end{array}$ & Norway & & $\begin{array}{c}\text { Norwegian Medicines } \\
\text { Agency }\end{array}$ & $\frac{\frac{\text { www.legem }}{\text { iddelverket. }}}{\text { no }}$ & & \\
\hline 35 & $\begin{array}{l}\text { OECD- } \\
\text { Eurostat }\end{array}$ & Poland & & $\begin{array}{c}\text { Office for } \\
\text { Registration of } \\
\text { Medicinal Products, } \\
\text { Medical } \\
\text { Devices and } \\
\text { Biocidal }\end{array}$ & $\frac{\text { www.urpl.go }}{\text { v.pl }}$ & $\begin{array}{l}\frac{\underline{\text { http://Www.w }}}{\text { ho.int/entity/s }} \\
\underline{\text { election }} \\
\frac{\text { medicines/co }}{\text { untry }} \\
\underline{\text { lists/Poland }} \\
\frac{\text { EML_2009.p }}{\underline{\text { df }}}\end{array}$ & 2009 \\
\hline 36 & $\begin{array}{l}\text { OECD- } \\
\text { Eurostat }\end{array}$ & Portugal & INFARMED & $\begin{array}{c}\text { National Authority } \\
\text { of } \\
\text { Medicines and } \\
\text { Health } \\
\text { Products } \\
\end{array}$ & $\frac{\frac{\mathrm{http}: / / \mathrm{www} .}{\text { infarmed.pt }}}{!}$ & & \\
\hline 37 & $\begin{array}{l}\text { OECD- } \\
\text { Eurostat }\end{array}$ & Romania & ANM & $\begin{array}{l}\text { National Medicines } \\
\text { Agency }\end{array}$ & $\begin{array}{r}\text { http://www.a } \\
\underline{\underline{\text { nm.r }}} \\
\underline{\text { o/en/ }} \\
\underline{\text { home }} \\
\underline{\text { html }}\end{array}$ & & \\
\hline 38 & $\begin{array}{l}\text { OECD- } \\
\text { Eurostat }\end{array}$ & $\begin{array}{l}\text { Russian } \\
\text { Federation }\end{array}$ & & $\begin{array}{c}\text { Federal Service for } \\
\text { Supervision of } \\
\text { Health }\end{array}$ & $\frac{\underline{\text { http://www.r }}}{\frac{\text { oszdravnadzo }}{\text { r.ru/ }}}$ & $\begin{array}{l}\text { Not available } \\
\text { online }\end{array}$ & \\
\hline 39 & $\begin{array}{l}\text { OECD- } \\
\text { Eurostat }\end{array}$ & Serbia & & $\begin{array}{c}\text { Medicines and } \\
\text { Medical } \\
\text { Devices Agency of } \\
\text { Serbia }\end{array}$ & $\frac{\underline{\text { http://www.al }}}{\underline{\text { ims.gov.rs/ }}}$ & $\begin{array}{l}\frac{\text { http://www.w }}{\text { ho.int/entity/s }} \\
\frac{\text { election }}{\text { medicines/co }} \\
\frac{\text { untry }}{\text { lists/SRB full }} \\
\frac{\text { y_overed_20 }}{\text { co.pdf }}\end{array}$ & 2010 \\
\hline 40 & $\begin{array}{l}\text { OECD- } \\
\text { Eurostat }\end{array}$ & Slovakia & SIDC & $\begin{array}{l}\text { State Institute for } \\
\text { Drug } \\
\text { Control }\end{array}$ & $\frac{\underline{\text { http://www.s }}}{\underline{\text { ukl.sk/en }}}$ & $\begin{array}{l}\frac{\underline{\text { http://www.w }}}{\text { ho.int/entity/s }} \\
\frac{\text { election }}{\text { medicines/co }} \\
\frac{\text { untry }}{\underline{\text { lists/Solvakia }}} \\
\underline{\text { EML }} \\
\underline{2010 . \mathrm{pdf}}\end{array}$ & 2010 \\
\hline 41 & $\begin{array}{l}\text { OECD- } \\
\text { Eurostat }\end{array}$ & Slovenia & JAZMP & $\begin{array}{l}\text { Public Agency for } \\
\text { Medical } \\
\text { Products and } \\
\text { Medical } \\
\text { Devices }\end{array}$ & $\begin{array}{l}\frac{\text { http://www.ja }}{\text { zmp.si/index. }} \\
\underline{\mathrm{php}} \\
\frac{? \mathrm{id}=}{\underline{56}}\end{array}$ & $\begin{array}{l}\frac{\mathrm{http}: / / \text { www.w }}{\text { ho.int/entity/s }} \\
\begin{array}{c}\text { election } \\
\text { medicines/co }\end{array} \\
\frac{\text { untry lists/svn }}{2010 . \mathrm{pdf}}\end{array}$ & 2010 \\
\hline 42 & OECD- & & AEMPS & Spanish Agency of & & & \\
\hline
\end{tabular}




\begin{tabular}{|c|c|c|c|c|c|c|c|}
\hline & Eurostat & Spain & & $\begin{array}{c}\text { Medicines and } \\
\text { Health Products }\end{array}$ & $\begin{array}{c}\frac{\text { www.aemps.g }}{\text { ob.es }} \\
\end{array}$ & & \\
\hline 43 & $\begin{array}{l}\text { OECD- } \\
\text { Eurostat }\end{array}$ & Sweden & MPA & $\begin{array}{c}\text { Medical Products } \\
\text { Agency }\end{array}$ & 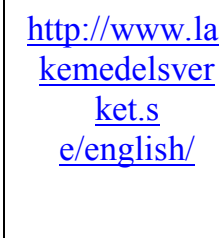 & 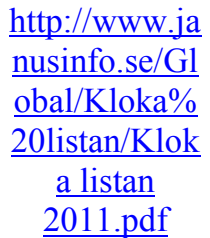 & 2011 \\
\hline 44 & $\begin{array}{l}\text { OECD- } \\
\text { Eurostat }\end{array}$ & Switzerland & & SwissMedic & $\begin{array}{r}\frac{\text { http://www.s }}{\text { wissmed }} \\
\frac{\text { ic.ch/ind }}{\text { ex.html? }} \\
\text { lang=en }\end{array}$ & & \\
\hline 45 & $\begin{array}{l}\text { OECD- } \\
\text { Eurostat }\end{array}$ & Turkey & $\mathrm{MOH}$ & $\begin{array}{c}\text { Ministry of Health of } \\
\text { Turkey, } \\
\text { General Directorate } \\
\text { of } \\
\text { Pharmaceuticals } \\
\text { and }\end{array}$ & 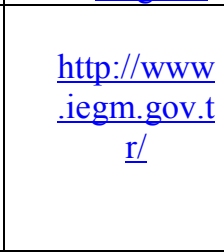 & & \\
\hline 46 & $\begin{array}{l}\text { OECD- } \\
\text { Eurostat }\end{array}$ & $\begin{array}{l}\text { UnitedKingd } \\
\text { om }\end{array}$ & MHRA & $\begin{array}{c}\text { Medicines and } \\
\text { Healthcare products } \\
\text { Regulatory Agency }\end{array}$ & $\frac{\frac{\text { http://www.m }}{\text { hra.gov.uk/in }}}{\underline{\text { dex }}}$ & & \\
\hline 47 & $\begin{array}{l}\text { OECD-- } \\
\text { Eurostat } \\
\text { Eurostat }\end{array}$ & $\begin{array}{l}\text { United } \\
\text { States }\end{array}$ & FDA & $\begin{array}{l}\text { Food and Drug } \\
\text { Administration }\end{array}$ & $\frac{\text { http://ww }}{\text { w.fda.gov/ }}$ & & \\
\hline
\end{tabular}

\section{PACIFIC ISLANDS ${ }^{1,2,7,8}$ :}

The Pacific Islands are the islands of the Pacific Ocean. Three major groups of islands in the Pacific Ocean are Polynesia, Micronesia and Melanesia.
Depending on the context, Pacific Islands may refer to countries and islands with common Austronesian origins, islands once or currently colonized, or Oceania.

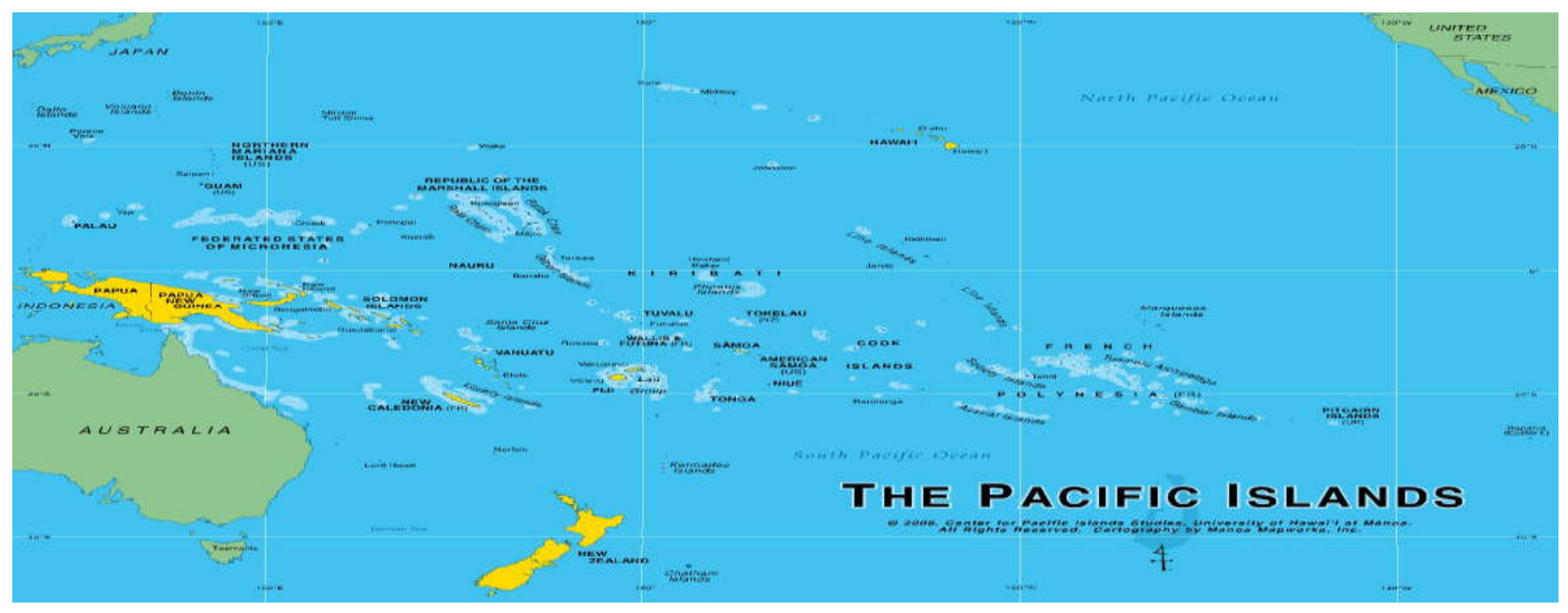




\begin{tabular}{|c|c|c|c|c|c|c|c|}
\hline Sr.No & $\begin{array}{l}\text { Regio } \\
\mathbf{n}\end{array}$ & Country & 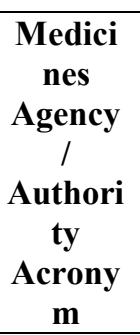 & $\begin{array}{l}\text { Medicines } \\
\text { Agency / } \\
\text { Authority }\end{array}$ & Website & $\begin{array}{l}\text { Essential Medicines } \\
\text { list }\end{array}$ & $\begin{array}{c}\text { Essential } \\
\text { Medicines list } \\
\text { year }\end{array}$ \\
\hline 1 & $\begin{array}{l}\text { Pacific } \\
\text { Islands }\end{array}$ & $\begin{array}{c}\text { American } \\
\text { Samoa }\end{array}$ & & & & & \\
\hline 2 & $\begin{array}{l}\text { Pacific } \\
\text { Islands }\end{array}$ & $\begin{array}{l}\text { Common } \\
\text { Wealth of } \\
\text { Mariana } \\
\text { Islands } \\
\end{array}$ & & & & & \\
\hline 3 & $\begin{array}{l}\text { Pacific } \\
\text { Islands }\end{array}$ & $\begin{array}{l}\text { Cook } \\
\text { Islands }\end{array}$ & & & & $\begin{array}{l}\text { http://www.who.int/enti } \\
\text { ty/se } \\
\text { lection_medicines/coun } \\
\text { try_lis } \\
\text { ts/cok_eml_2007.pdf }\end{array}$ & 2007 \\
\hline 4 & $\begin{array}{l}\text { Pacific } \\
\text { Islands }\end{array}$ & $\begin{array}{c}\text { Federal } \\
\text { States of } \\
\text { Micronesi } \\
\text { a }\end{array}$ & & & & & \\
\hline 5 & $\begin{array}{l}\text { Pacific } \\
\text { Islands }\end{array}$ & Fiji & IRA & $\begin{array}{l}\text { Inspectorate } \\
\text { \&Regulator } \\
\text { y Authority }\end{array}$ & 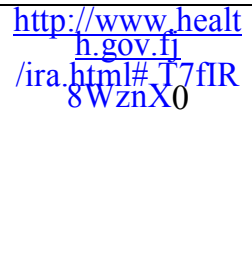 & $\begin{array}{l}\text { http://www.who.int/e } \\
\underline{\text { ntity/se }} \\
\text { lection_medicines/co } \\
\text { untry_lis } \\
\text { ts/fji_formulary_200 } \\
\text { 6.pdf }\end{array}$ & 2006 \\
\hline 6 & $\begin{array}{l}\text { Pacific } \\
\text { Islands }\end{array}$ & $\begin{array}{c}\text { French } \\
\text { Polynesia }\end{array}$ & & & & & \\
\hline 7 & $\begin{array}{l}\text { Pacific } \\
\text { Islands }\end{array}$ & Guam & & & & & \\
\hline 8 & $\begin{array}{l}\text { Pacific } \\
\text { Islands }\end{array}$ & Kiribati & & & & $\begin{array}{l}\text { http://ww.who.int/entity } \\
\text { /selection } \\
\text { medicines/country } \\
\text { lists/kir EDL 2009.pdf }\end{array}$ & 2009 \\
\hline 9 & $\begin{array}{l}\text { Pacific } \\
\text { Islands }\end{array}$ & $\begin{array}{l}\text { Marshall } \\
\text { Islands }\end{array}$ & & & & 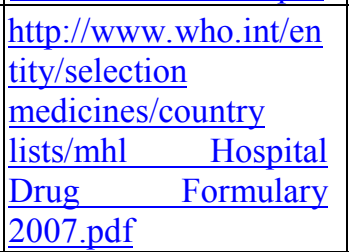 & 2007 \\
\hline 10 & $\begin{array}{l}\text { Pacific } \\
\text { Islands }\end{array}$ & Nauru & & & & $\begin{array}{l}\frac{\text { http://who.int/entity/sel }}{\text { ection }} \\
\frac{\text { medicines/country lists }}{\text { nru 2010.pdf }}\end{array}$ & 2010 \\
\hline 11 & Pacific & New & & & & & \\
\hline
\end{tabular}




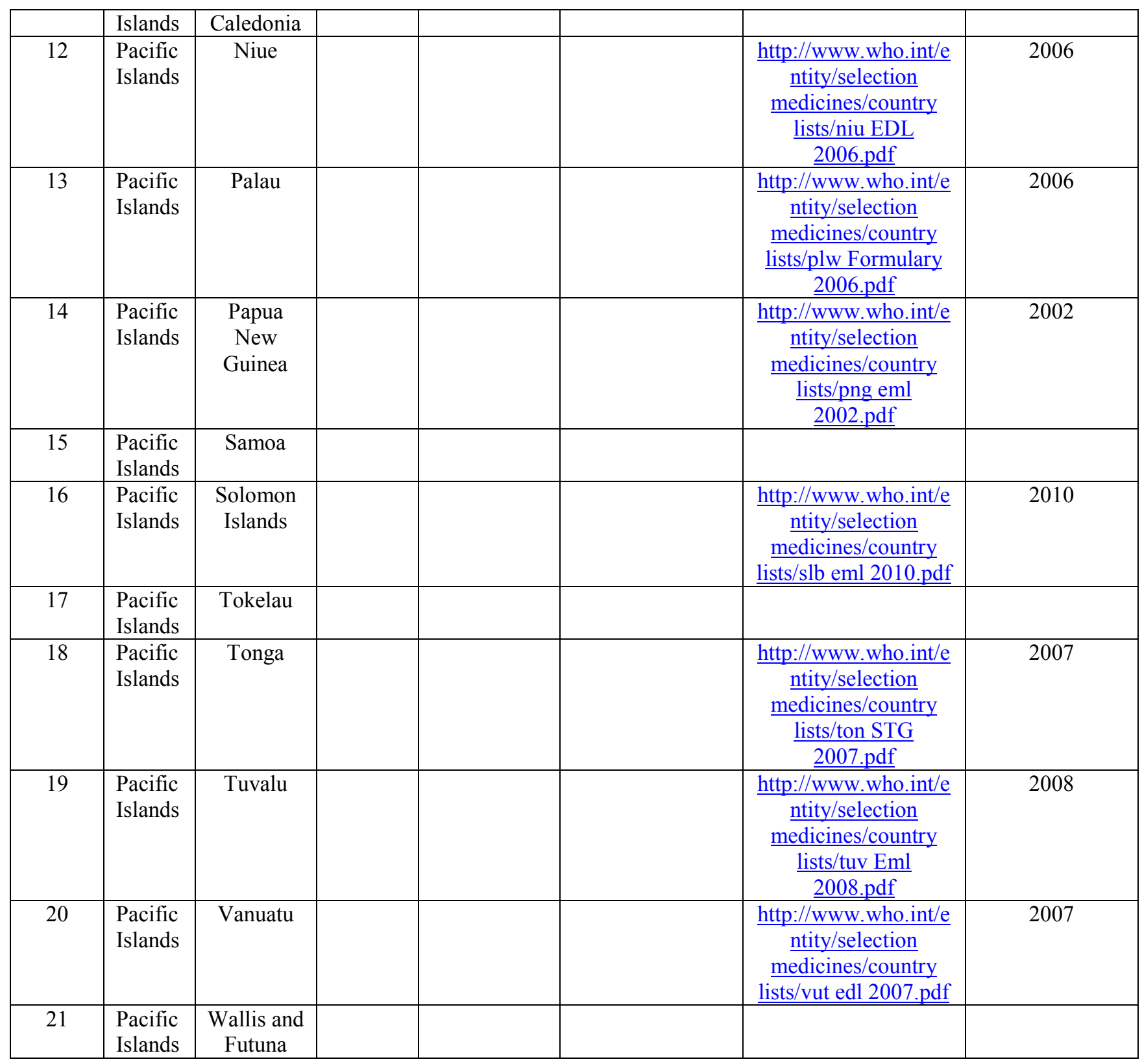

\section{SINGLETON COUNTRIES ${ }^{1,2,9,10}$ :}

Iran and Georgia have had relations for millennia, although official diplomatic relations between the two nations in the 20th century were established on
May 15, 1992. Georgia is represented by its embassy in Tehran, while Iran has its representative embassy in Tbilisi. Iran is an important trade partner of Georgia. 


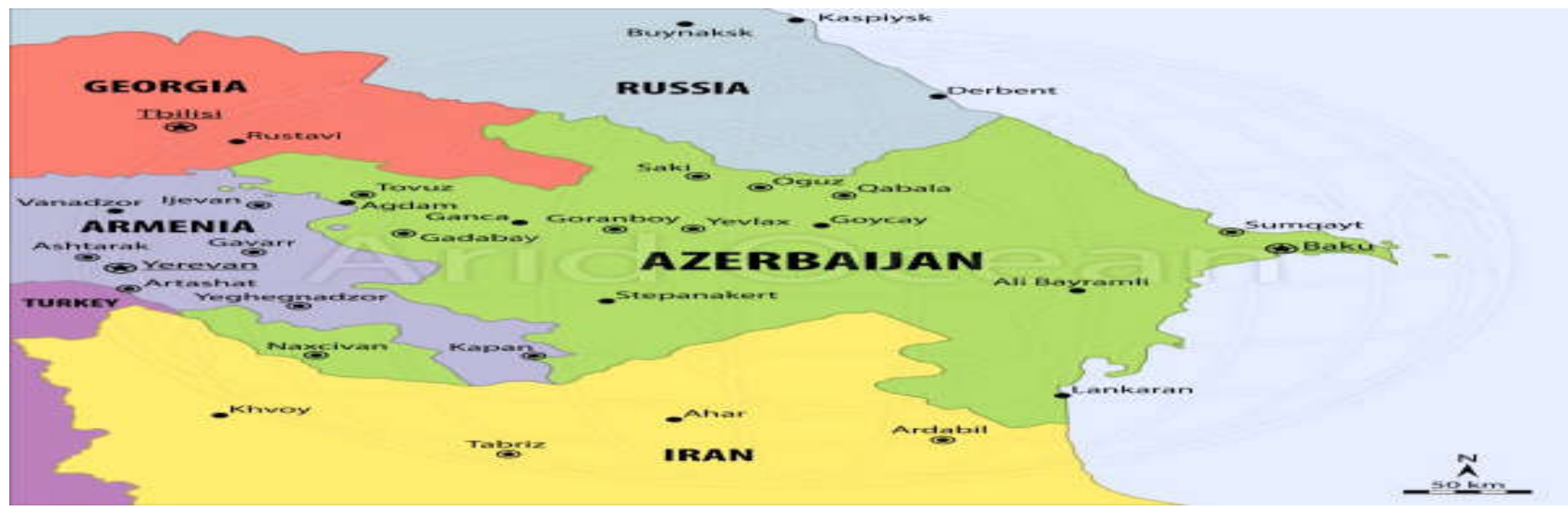

\begin{tabular}{|c|c|c|c|c|c|c|c|}
\hline Sr.No & Region & Country & $\begin{array}{c}\text { Medicines } \\
\text { Agency/ } \\
\text { Authority } \\
\text { Acronym }\end{array}$ & $\begin{array}{l}\text { Medicines } \\
\text { Agency / } \\
\text { Authority }\end{array}$ & Website & $\begin{array}{c}\text { Essential Medicines } \\
\text { list }\end{array}$ & $\begin{array}{c}\text { Essential } \\
\text { Medicin } \\
\text { es list } \\
\text { year }\end{array}$ \\
\hline 1 & $\begin{array}{l}\text { Singleton } \\
\text { Countries }\end{array}$ & Iran & MOHME & $\begin{array}{l}\text { Ministry } \\
\text { of Health } \\
\text { and } \\
\text { Medical } \\
\text { Education }\end{array}$ & $\begin{array}{l}\text { http://www.moh.gov } \\
. \text { iq/english/index.php }\end{array}$ & $\begin{array}{l}\frac{\text { http://www.who.int/e }}{\text { ntit }} \\
\frac{\text { y/selection_medicin }}{\text { es/co }} \\
\frac{\text { untry_lists/irn_EDL }}{\text { 2009. pdf }}\end{array}$ & 2009 \\
\hline 2 & $\begin{array}{l}\text { Singleton } \\
\text { Countries }\end{array}$ & Georgia & GDNA & $\begin{array}{l}\text { Georgia } \\
\text { Drugs and } \\
\text { Narcotics } \\
\text { Agency }\end{array}$ & $\begin{array}{c}\frac{\text { http://gdna.georgia. }}{\text { gov/02/gdna/home/ }} \\
\frac{0,2803,132319894,0}{0 . h t m l}\end{array}$ & $\begin{array}{l}\frac{\underline{\text { http://apps.who.int } / \mathrm{m}}}{\underline{\text { edic }}} \\
\frac{\underline{\text { inedocs } / \text { documents } / \mathrm{s}}}{\frac{1902}{\text { 5en/s19025en. }}} \\
\text { pdf }\end{array}$ & 2007 \\
\hline
\end{tabular}

\section{WESTERN ASIA ${ }^{1,2,11}$ :}

Western Asia is located directly south of Eastern Europe. The region is surrounded by seven major seas; the Aegean Sea, the Black Sea, the Caspian Sea, the Persian Gulf, the Arabian Sea, the Red Sea, and the Mediterranean Sea.
To the north, the region is delimited from Europe by the Caucasus Mountains, to the southwest, it is delimited from Africa by the Isthmus of Suez, while to the east, the region adjoins Central Asia and South Asia.

The Dasht-e Kavir and Dasht-e Lut deserts in eastern Iran naturally delimit the region somewhat from Asia itself. 


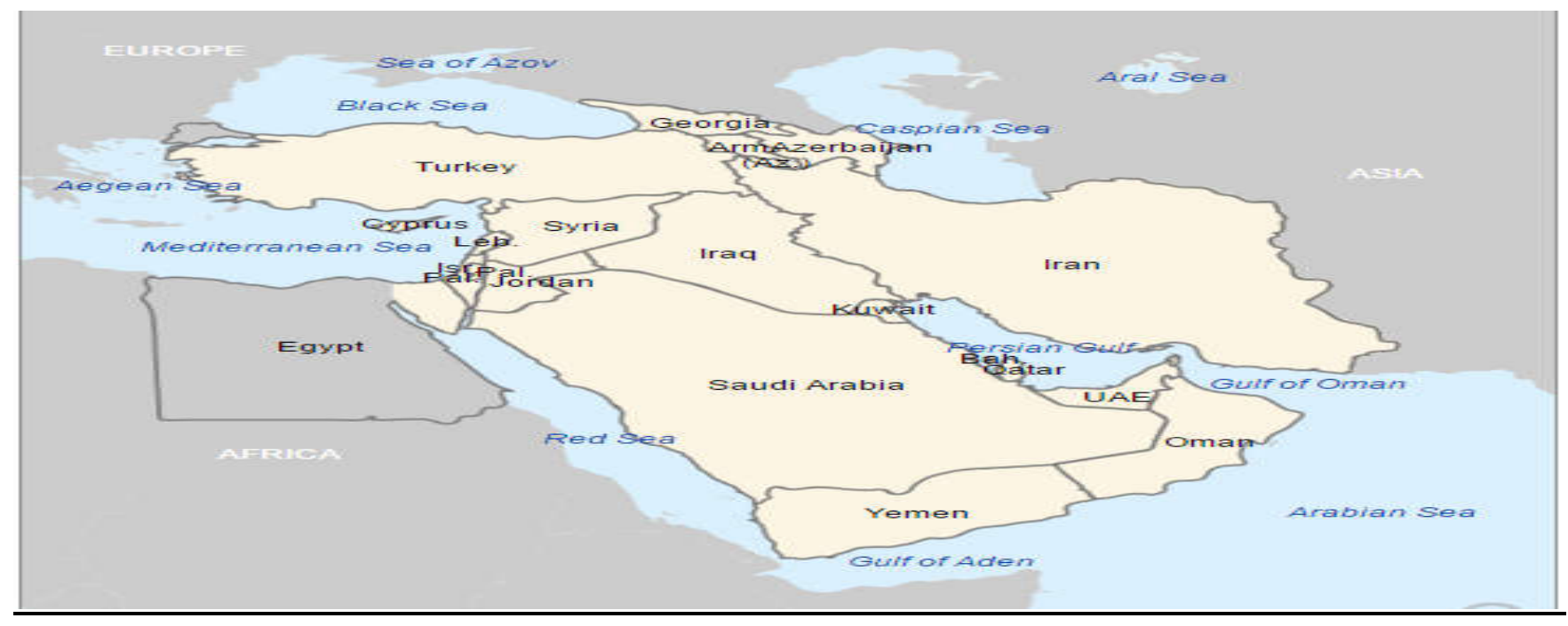

\begin{tabular}{|c|c|c|c|c|c|c|c|}
\hline $\begin{array}{l}\text { Sr. } \\
\text { No }\end{array}$ & Region & $\begin{array}{c}\text { Countr } \\
\mathbf{y}\end{array}$ & $\begin{array}{c}\text { Medicines } \\
\text { Agency/ } \\
\text { Authority } \\
\text { Acronym }\end{array}$ & $\begin{array}{l}\text { Medicines } \\
\text { Agency / } \\
\text { Authority }\end{array}$ & Website & Essential Medicines list & $\begin{array}{l}\text { Essential } \\
\text { Medicine } \\
\text { s list year }\end{array}$ \\
\hline 1 & $\begin{array}{l}\text { Western } \\
\text { Asia }\end{array}$ & Bahrain & & $\begin{array}{c}\text { Ministry of } \\
\text { Health:Pharmac } \\
\text { eutical and } \\
\text { Drug Control } \\
\text { Directory }\end{array}$ & $\begin{array}{l}\frac{\text { https://www.m }}{\text { oic.gov.bh/Mo }} \\
\frac{\text { ICEn/M }}{\text { olC+Centers/B }} \\
\frac{\text { ahrainInvestors }}{\text { Center/Li }} \\
\frac{\text { censing+Autho }}{\text { rities/Ministry }} \\
\frac{\text { OfHealth/ }}{\text { PharmacyandD }} \\
\frac{\text { rug+ontrolDire }}{\text { ctorate/ }}\end{array}$ & $\begin{array}{l}\frac{\text { http://apps.who.int/medi }}{\text { cinedoc }} \\
\underline{\text { s/documents/s19536en/s }} \\
\underline{19536 \mathrm{e} \text { n.pdf }}\end{array}$ & 2009 \\
\hline 2 & $\begin{array}{l}\text { Western } \\
\text { Asia }\end{array}$ & Egypt & EDA & $\begin{array}{c}\text { Egyptian Drug } \\
\text { Authority }\end{array}$ & $\underline{\underline{\text { http://www.eda }}}$ & $\begin{array}{l}\frac{\underline{\text { http://www.who.int/entit }}}{\mathrm{y} / \text { select }} \\
\frac{\text { ion_medicines/country_1 }}{\frac{\text { ists/EGY }}{\text { EML 2006.p }}} \\
\frac{\text { df }}{2}\end{array}$ & 2006 \\
\hline 3 & $\begin{array}{c}\text { Western } \\
\text { Asia }\end{array}$ & Iraq & & $\begin{array}{l}\text { Directorete of } \\
\text { Technical } \\
\text { Affairs in the } \\
\text { Ministry of } \\
\text { Health }\end{array}$ & & $\begin{array}{l}\frac{\text { http://apps.who.int/medi }}{\text { cinedoc }} \\
\frac{\text { s/documents/s17416e/s1 }}{\underline{7416 \mathrm{e} . p} \underline{\mathrm{df}}}\end{array}$ & 2010 \\
\hline 4 & $\begin{array}{c}\text { Western } \\
\text { Asia }\end{array}$ & Jordan & JFDA & $\begin{array}{c}\text { Jordan Food } \\
\text { and Drug }\end{array}$ & $\frac{\text { http://www.jfd }}{\text { a.jo/Default.as }}$ & $\frac{\text { http://apps.who.int/medi }}{\underline{\text { cinedoc }}}$ & 2011 \\
\hline
\end{tabular}




\begin{tabular}{|c|c|c|c|c|c|c|c|}
\hline & & & & Administration & $\mathrm{px}$ & $\frac{\text { s/documents/s19466en/s }}{19466 \mathrm{e} \text { n.pdf }}$ & \\
\hline 5 & $\begin{array}{l}\text { Western } \\
\text { Asia }\end{array}$ & Kuwait & & & & & \\
\hline 6 & $\begin{array}{l}\text { Western } \\
\text { Asia }\end{array}$ & Oman & $\mathrm{MOH}$ & $\begin{array}{l}\text { Ministry of } \\
\text { Health }\end{array}$ & $\begin{array}{l}\frac{\text { http://www.mo }}{\text { h.gov.om/en/n }} \\
\text { v_menu.php?o } \\
=\text { pharma/regul } \\
\underline{\text { a tion.htm }}\end{array}$ & $\begin{array}{l}\frac{\text { http://www.who.int/entit }}{\mathrm{y} / \text { select }} \\
\frac{\text { ion_medicines/country 1 } 1}{\text { ists/omn }} \\
\text { EDL 2009.pdf }\end{array}$ & 2009 \\
\hline 7 & $\begin{array}{l}\text { Western } \\
\text { Asia }\end{array}$ & Qatar & & $\begin{array}{l}\text { Supreme } \\
\text { council of } \\
\text { Health }\end{array}$ & $\begin{array}{c}\frac{\underline{\mathrm{http}: / / \mathrm{www} . \mathrm{sch}}}{\text { gov.qa/sch/E }} \\
\frac{\underline{\mathrm{n} / \mathrm{index} . j \mathrm{sp} ? \mathrm{CS}}}{\mathrm{RT}=17710338} \\
\frac{41318}{\underline{73616}} \\
\underline{3}\end{array}$ & & \\
\hline 8 & $\begin{array}{l}\text { Western } \\
\text { Asia }\end{array}$ & $\begin{array}{c}\text { Saudi } \\
\text { Arabia }\end{array}$ & SFDA & $\begin{array}{l}\text { Saudi food and } \\
\text { Drug Authority }\end{array}$ & $\frac{\text { http://www.s }}{\text { fda.gov.sa/ }}$ & Not Available online. & \\
\hline 9 & $\begin{array}{l}\text { Western } \\
\text { Asia }\end{array}$ & Sudan & NMPB & $\begin{array}{c}\text { National } \\
\text { Medicines and } \\
\text { Poisons Board }\end{array}$ & $\frac{\text { http://www.n }}{\underline{\text { mpb.gov.sd }}}$ & $\begin{array}{l}\frac{\text { http://www.who.int/entit }}{y / \text { select }} \\
\frac{\text { ion_medicines/country 1 }}{\text { ists/sdn_eml 2007.pdf }}\end{array}$ & 2007 \\
\hline 10 & $\begin{array}{l}\text { Western } \\
\text { Asia }\end{array}$ & $\begin{array}{c}\text { United } \\
\text { Arab } \\
\text { Emirate } \\
\text { s }\end{array}$ & $\mathrm{MOH}$ & $\begin{array}{l}\text { Ministry of } \\
\text { Health }\end{array}$ & $\begin{array}{r}\frac{\text { http://www.mo }}{\text { h.gov.ae/e }} \\
\frac{\underline{\mathrm{n} / \mathrm{Pa}}}{\text { ge } 431 . a \mathrm{se}} \\
\underline{\mathrm{px}}\end{array}$ & & \\
\hline 11 & $\begin{array}{l}\text { Western } \\
\text { Asia }\end{array}$ & $\begin{array}{c}\text { West } \\
\text { bank \& } \\
\text { Gaza }\end{array}$ & & & & & \\
\hline 12 & $\begin{array}{l}\text { Western } \\
\text { Asia }\end{array}$ & Yemen & & $\begin{array}{l}\text { Supreme Board } \\
\text { for Drugs \& } \\
\text { Medical } \\
\text { Appliances }\end{array}$ & $\frac{\text { http://www.sbd }}{\text {-ye.org/ }}$ & $\begin{array}{l}\frac{\text { http://www.who.int/entit }}{\mathrm{y} / \text { select }} \\
\frac{\text { ion_medicines/country 1 } 1}{\text { ists/yem }} \\
\text { EDL FINAL 2007.pdf }\end{array}$ & 2007 \\
\hline
\end{tabular}

Non- ICP Countries ${ }^{1,2}$ :

\begin{tabular}{|c|c|c|c|c|c|c|c|}
\hline $\begin{array}{c}\text { Sr.N } \\
\text { o }\end{array}$ & Region & Country & $\begin{array}{c}\text { Medicin } \\
\text { es } \\
\text { Agency/ } \\
\text { Authorit } \\
\text { y } \\
\text { Acrony } \\
\text { m }\end{array}$ & $\begin{array}{c}\text { Medicines } \\
\text { Agency / } \\
\text { Authority }\end{array}$ & Website & $\begin{array}{c}\text { Essential } \\
\text { Medicines list }\end{array}$ & $\begin{array}{l}\text { Essential } \\
\text { Medicine } \\
\text { s list year }\end{array}$ \\
\hline 1 & Wester & Afghanistan & & & & http://www.who.int/e & 2007 \\
\hline
\end{tabular}




\begin{tabular}{|c|c|c|c|c|c|c|c|}
\hline & n Asia & & & & & \begin{tabular}{|c|}
$\underline{\text { ntity/selection }}$ \\
medicines/country list \\
/AFG EML 07.pdf \\
AF E
\end{tabular} & \\
\hline 2 & $\begin{array}{l}\text { Wester } \\
\text { n Asia }\end{array}$ & Lebanon & MOPH & $\begin{array}{c}\text { Ministry of } \\
\text { Public Health }\end{array}$ & $\frac{\text { http://ww.moph.gov.lb/ }}{\underline{\text { Pages/Home.aspx }}}$ & $\begin{array}{l}\frac{\text { http://apps.who.int/ }}{\text { medicinedocs/docu }} \\
\frac{\text { ments/s19535en/s1 }}{\text { 9535en.pdf }}\end{array}$ & 2010 \\
\hline 3 & $\begin{array}{l}\text { Asia \& } \\
\text { Pacific }\end{array}$ & DRP Korea & & & & $\frac{\text { http://ww.who.int/e }}{\text { ntity/selection }}$ & \\
\hline 4 & $\begin{array}{l}\text { Asia \& } \\
\text { Pacific }\end{array}$ & $\begin{array}{l}\text { Democratic } \\
\text { Re Public of } \\
\text { Timor Leste }\end{array}$ & & & & $\begin{array}{l}\frac{\text { http://www.who.int }}{\text { lentity/selection }} \\
\frac{\text { medicines/country }}{\underline{\text { lists/tls eml }}} \\
\underline{2004 . p d f}\end{array}$ & 2004 \\
\hline 5 & $\begin{array}{c}\text { OECD } \\
- \\
\text { Eurosta } \\
t\end{array}$ & Andorra & $\mathrm{MOH}$ & $\begin{array}{l}\text { Ministry of } \\
\text { Health and } \\
\text { Welfare }\end{array}$ & $\frac{\text { http://www.salutibenest }}{\underline{\text { ar.ad }}}$ & & \\
\hline 6 & $\begin{array}{l}\text { OECD- } \\
\text { Eurosta } \\
\mathrm{t}\end{array}$ & Kosovo & KMA & $\begin{array}{l}\text { Kosovo } \\
\text { Medicines } \\
\text { Agency }\end{array}$ & http://www.k-ma.org & & \\
\hline 7 & $\begin{array}{c}\text { OECD- } \\
\text { Eurosta } \\
t\end{array}$ & $\begin{array}{c}\text { Liechtenstei } \\
n\end{array}$ & & $\begin{array}{c}\text { Office of } \\
\text { Health - } \\
\text { Department of } \\
\text { Pharmaceutica } \\
\text { ls } \\
\end{array}$ & www.llv.li & & \\
\hline 8 & $\begin{array}{l}\text { Wester } \\
\text { n Asia }\end{array}$ & $\begin{array}{l}\text { Syrian Arab } \\
\text { Republic }\end{array}$ & & & & $\begin{array}{l}\frac{\text { http://www.who.int }}{\text { /selection }} \\
\frac{\text { medicines/country }}{\frac{\text { lists/SYRNML }}{\text { 08.pdf }}}\end{array}$ & 2008 \\
\hline 9 & $\begin{array}{l}\text { Wester } \\
\mathrm{n} \text { Asia }\end{array}$ & Somalia & & & & $\begin{array}{l}\frac{\text { http://www.who.int }}{\text { /selection }} \\
\text { medicines/country } \\
\underline{\text { lists/som }} \\
\underline{\text { stg2007.pdf }}\end{array}$ & 2006 \\
\hline 10 & Africa & Eritrea & & $\begin{array}{l}\text { Department of } \\
\text { Regulatory } \\
\text { Services }\end{array}$ & & $\begin{array}{l}\frac{\text { http://www.who.int }}{\text { /entity/selection }} \\
\frac{\text { medicines/country }}{\frac{\text { lists/eri eml }}{2010 . p d f}}\end{array}$ & 2010 \\
\hline
\end{tabular}

\section{CONCLUSION:}

In this article the world was divided in as ICP and NON-ICP countries. In these countries are given based on region wide. This article gives the information about individual country regulatory authority and its official websites to search about any marketing authorization procedures, their guidelines ,to check the market need about drugs and cosmetics. 
This articles also gives information about essential medical list and their published year. This article reduces the time of people to search websites, authorities of different countries. In this article we clearly mention the medical list any one can search

\section{REFERENCES:}

1. https://www.state.gov/misc/list/

2. http://www.who.int/medicines/areas/quality saf ety/regulation legislation/icdra/PL2-

A ProgressReport_WPRO.pdf

3. https://en.wikipedia.org/wiki/Commonwealth_o f Independent States

4. https://images.search.yahoo.com/yhs/search; yl $\underline{t=A w r T c c g g f R I Z E G 4 A ~ d w P x Q t . ? p=h o w ~}+$ man $\mathrm{y}+$ countries + in + commonwealth + of + independe

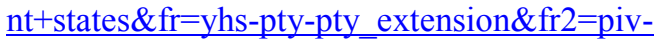
web\&hspart=pty\&hsimp=yhspty extension\#id=2\&iurl=http $\% 3 \mathrm{~A} \% 2 \mathrm{~F} \% 2 \mathrm{FW}$ ww.lib.utexas.edu $\% 2$ Fmaps $\% 2$ Fcommonwealt $\underline{\mathrm{h} \% 2 \mathrm{Fcommonwealth.jpg \& action}=\text { click }}$

5. https://images.search.yahoo.com/yhs/search; yl $\mathrm{t}=\mathrm{A} 86 . J y U L h R l Z u D 8 A i 4 w P x Q t . ? \mathrm{p}=$ how + man $\mathrm{y}+$ countries + in + Latin + America $+\% 26+$ Caribbe their medicines according their requirements. It will be helpful to all industries of different countries to follow the guidelines for the marketing authorization of their products.

an\%3A\&fr=yhs-pty-pty_extension\&fr $2=$ pivweb\&hspart $=$ pty\&hsimp $=$ yhs -

pty_extension\#id=0\&iurl $=\mathrm{http} \% 3 \mathrm{~A} \% 2 \mathrm{~F} \% 2 \mathrm{Fw}$ orldartswest.org $\% 2 \mathrm{FAssets} \% 2 \mathrm{FLocations} \% 2 \mathrm{~F}$ Latin\%2520America.gif\&action=click

6. https://en.wikipedia.org/wiki/Latin_America

7. https://en.wikipedia.org/wiki/Pacific Islands

8. http://www.japanfocus.org/data/sm_pacific_we b map.jpg

9. https://pbs.twimg.com/media/CaC3V3ZWQAA 7qSG.jpg:large

10. https://en.wikipedia.org/wiki/Georgia $\% \mathrm{E} 2 \% 80$ \%93Iran_relations

11. https://en.wikipedia.org/wiki/Western_Asia

12. http://www.worldometers.info/geography/howmany-countries-in-africa/

13. https://en.wikipedia.org/wiki/Asia-Pacific

14. http://apcss.org/about-2/ap-countries/ 
Indian Research Journal of Pharmacy and Science;). Yoshasri et.al.

[June'17]

Ind Res J Pharm \& Sci|2017: June.:4(2) 